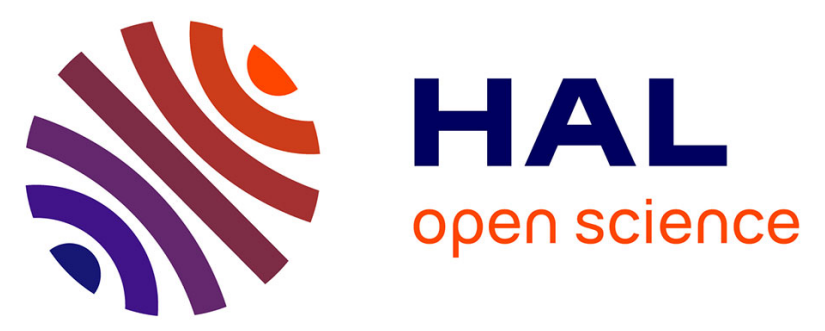

\title{
De la formation géologique à la tranchée: trouver et comprendre les sites archéologiques menacés par les travaux d'aménagement du territoire
}

Patrice Wuscher, Christophe Jorda, Quentin Borderie, Nathalie Schneider, Laurent Bruxelles

\section{To cite this version:}

Patrice Wuscher, Christophe Jorda, Quentin Borderie, Nathalie Schneider, Laurent Bruxelles. De la formation géologique à la tranchée: trouver et comprendre les sites archéologiques menacés par les travaux d'aménagement du territoire. Archimède: archéologie et histoire ancienne, 2020, 7, pp.158175. 10.47245/archimede.0007.act.05 . halshs-02893418

\section{HAL Id: halshs-02893418 \\ https://shs.hal.science/halshs-02893418}

Submitted on 8 Jul 2020

HAL is a multi-disciplinary open access archive for the deposit and dissemination of scientific research documents, whether they are published or not. The documents may come from teaching and research institutions in France or abroad, or from public or private research centers.
L'archive ouverte pluridisciplinaire HAL, est destinée au dépôt et à la diffusion de documents scientifiques de niveau recherche, publiés ou non, émanant des établissements d'enseignement et de recherche français ou étrangers, des laboratoires publics ou privés. 


\section{ARCHIMÈDE N N 7}

\section{DOSSIER THÉMATIQUE : GESTES RITUELS. DE LA TRACE À L'INTERPRÉTATION}

\section{ACTUALITÉ DE LA RECHERCHE GÉOSCIENCES ET ARCHÉOLOGIE : INTERACTIONS, COMPLÉMENTARITÉS ET PERSPECTIVES}

114 Bruno GAVAZZI

Heureuses rencontres. Vers le développement d'approches intégrées en géosciences et archéologie

119 Jean-Paul BRAVARD

Dialogue interdisciplinaire : de I'unité stratigraphique aux interactions culture-environnement

129 Ferréol SALOMON

Les origines d'Ostie : quelles interactions avec la dynamique d'embouchure? (Delta du Tibre, Italie)

141 Quentin BORDERIE, Rowena Y. BANERJEA, Stéphane BONNET, Yannick DEVOS, Cristiano NICOSIA, Christophe PETIT, Ferréol SALOMON, Nathalie SCHNEIDER, Barbora WOUTERS, \& Patrice WUSCHER Géoarchéologies des contextes urbains : mieux comprendre les modalités de l'artificialisation des géosystèmes

158 Patrice WUSCHER, Christophe JORDA, Quentin BORDERIE, Nathalie SCHNEIDER \& Laurent BRUXELLES De la formation géologique à la tranchée : trouver et comprendre les sites archéologiques menacés par les travaux d'aménagement du territoire

176 Morgan MILLET \& Michel GUÉLAT

Les vestiges antiques de Rennaz-Noville (Vaud, Suisse) et leur contexte sédimentaire : nouvelles évidences de l'écroulement du Tauredunum

188 Théophile PIAU, François BÉTARD, Fabienne DUGAST, Gilles ARNAUD-FASSETTA \& Vincent VIEL Dynamique géomorphologique holocène et occupation humaine dans le bassin-versant de l'Eure (Bassin de Paris, France) : potentiels d'une approche géoarchéologique multiscalaire et diachronique

205 Dominique SCHWARTZ, Vincent ROBIN, Pierre ADAM, Philippe SCHAEFFER, Anne GEBHARDT, Pierre-Alexis HERRAULT, Benjamin KELLER, Daniele DAPIAGGI, Claire STEVENEL, Maxime THISS, Martine TRAUTMANN \& Damien ERTLEN

Les géosciences au service de l'archéologie agraire. Une étude de cas sur les rideaux de culture de Goldbach (68)

217 Étienne MANTEL, Stéphane DUBOIS, Jonas PARÉTIAS, Victor VISQUESNEL-SCHLOSSER, Corentin VOISIN, Bruno GAVAZZI \& Matthieu RICHARD

Étudier I'occupation d'une ville : les enjeux du PCR « Topographie générale et insertion territoriale de l'agglomération antique de Briga »

231 Charlène MOREL

Un modèle pour comprendre l'influence de l'état de la recherche, des processus post-déposition et de l'attractivité sur la découverte des sites archéologiques dans le Kochersberg (Bas-Rhin, Alsace, Grand-Est, France)

241 Lizzie SCHOLTUS

Spatialisation des découvertes, modélisation du passé. L'informatique au service de l'archéologie

254 Guillaume HULIN \& François-Xavier SIMON

Inrap et géophysique : vers une approche raisonnée

260 Hugo REILLER, Matthieu FUCHS, \& Bruno GAVAZZI

Approche multi-méthodes expérimentale pour l'étude d'un site d'occupation romaine et médiévale à Horbourg-Wihr

272 François-Xavier SIMON, Julien GUILLEMOTEAU, Guillaume HULIN, Joachim RIMPOT, Julien THIESSON \& Alain TABBAGH

De nouvelles perspectives pour les applications des méthodes électromagnétiques basse fréquence en archéologie

283 Rémy WASSONG \& Bruno GAVAZZI

Apport des prospections magnétiques haute résolution à la compréhension d'un habitat protohistorique : l'exemple du site de hauteur fortifié du Maimont 


\title{
DE LA FORMATION GÉOLOGIQUE À LA TRANCHÉE : TROUVER ET COMPRENDRE LES SITES ARCHÉOLOGIQUES MENACÉS PAR LES TRAVAUX D'AMÉNAGEMENT DU TERRITOIRE
}

\author{
Patrice WUSCHER ${ }^{1,2, *}$, Christophe JORDA ${ }^{3,4}$, Quentin BORDERIE ${ }^{4,5}$, \\ Nathalie SCHNEIDER ${ }^{3,2}$, Laurent BRUXELLES ${ }^{3,6}$ \\ ${ }^{1}$ Archéologie Alsace \\ ${ }^{2}$ Laboratoire Image Ville Environnement, UMR 7362 Université de Strasbourg/CNRS \\ ${ }^{3}$ INRAP \\ ${ }^{4}$ Archéologie des Sociétés Méditerranéennes, Université Paul Valéry/CNRS \\ ${ }^{4}$ Département d'Eure-et-Loir \\ ${ }^{5}$ UMR 7041 Arscan «Archéologies Environnementales», Paris I, Paris Nanterre, CNRS et Ministère de la Culture \\ ${ }^{6}$ Laboratoire Traces - UMR 5608, Université Toulouse Jean Jaurès \\ *contact : patrice.wuscher@archeologie.alsace
}

\section{RÉSUMÉ}

Les sciences de la terre ont été mises à contribution dès les premières recherches paléolithiques au XIX siècle pour trouver les sites archéologiques et participer à l'étude de l'adaptation des sociétés du passé aux changements environnementaux. Elles ont pris un rôle important dans les dispositifs d'archéologie préventive élaborés à partir des années 1980 pour sauvegarder les vestiges menacés par les travaux d'aménagement du territoire. La présente contribution vise à illustrer la démarche pluridisciplinaire à l'œuvre depuis plusieurs décennies, depuis les études documentaires et cartographiques jusqu'à l'ouverture de tranchées de diagnostic. Elle se propose également de montrer quelques apports de la géoarchéologie et de l'archéologie préventive aux questions géomorphologiques, à la reconstitution des dynamiques des paysages et à l'impact de l'Homme sur l'environnement. Les exemples présentés ont été choisis dans des contextes géographiques différents, le sud de la France,

MOTS-CLÉS

Géomorphologie, géoarchéologie, archéologie préventive, diagnostics archéologiques, paysages. le bassin de Paris et l'Alsace, et s'échelonnent du Paléolithique à l'époque moderne pour illustrer les potentiels de la démarche qui gagnerait à être appliquée de façon plus systématique.
Earth sciences were used since the earliest paleolithic research in the 19th century to find archaeological sites and to study the adaptation of past societies to environmental changes. They took an important role in the preventive archaeology systems developed from the 1980 s to save the remains threatened by territory development. This contribution aims to illustrate the multidisciplinary approach developed over several decades, from documentary and cartographic studies to the opening of test pits. It also intends to show some contributions of geoarchaeology and preventive archaeology to geomorphological questions, to the reconstruction of landscape dynamics and to the question of human impact on the environment. The examples presented were chosen in different geographical contexts, in the south of France, Paris basin and Alsace, from the paleolithic to the modern era, to illustrate the potential of the approach which would benefit from being applied more systematically.
KEYWORDS

Geomorphology, geoarchaeology, rescue archaeology, test pits, landscapes. 


\section{INTRODUCTION}

En reconstituant la paléogéographie, les paléoenvironnements et les stratégies d'adaptation des sociétés du passé aux changements environnementaux, les disciplines archéologiques apportent des éléments de réflexion aux enjeux actuels de la raréfaction des ressources et du réchauffement climatique. Ces travaux démarrent par la recherche de sites archéologiques et la constitution d'un corpus suffisamment étoffé de données. Les sciences de la terre qui permettent notamment de comprendre l'empilement des strates et des paléosols dans les différents compartiments des bassins-versants, des versants aux littoraux, ont depuis longtemps été mises à contribution lors de cette étape fondamentale, depuis les premières recherches paléolithiques dans les gravières de la Somme au XIX siècle [1]. Dans le contexte de destruction effrénée de vestiges archéologiques par les travaux d'aménagement du territoire des dernières décennies, ces disciplines ont pris un rôle central dans les dispositifs de cartographie et de fouilles préventives des vestiges archéologiques élaborés à partir des années 1980 .

Ainsi, la géomorphologie est associée aux premières opérations d'archéologie préventive [2] et des cartes géomorphologiques et des campagnes de sondages à la tarière sont commanditées pour participer à la détection des vestiges archéologiques menacés par des travaux d'aménagement [3]. En parallèle, des sondages à la pelle mécanique sur 5 à $10 \%$ des surfaces aménagées se sont imposés pour détecter les sites archéologiques, tandis que certaines carrières faisaient l'objet jusque dans les années 2000 de suivi de décapage sur l'intégralité de leur emprise [4]. La loi de 2001 a systématisé le dispositif d'archéologie préventive basé sur les diagnostics et les fouilles. Pour les diagnostics, la méthode de sondages autour de $10 \%$ des terrains prospectés est devenue la norme, mais est généralement fonction de la puissance du recouvrement sédimentaire. Depuis, cette méthode a souvent été discutée [5] et est régulièrement remise en question, par exemple en 2014 avec le projet de loi relatif à la simplification de la vie des entreprises, dont une des mesures, la mesure $n^{\circ} 14$, non retenue dans la loi approuvée, prévoyait la possibilité de remplacer les ouvertures mécaniques par des prospections géophysiques.

Le présent article vise à illustrer, à partir de quelques exemples, la nécessité qu'il y a à combiner lecture

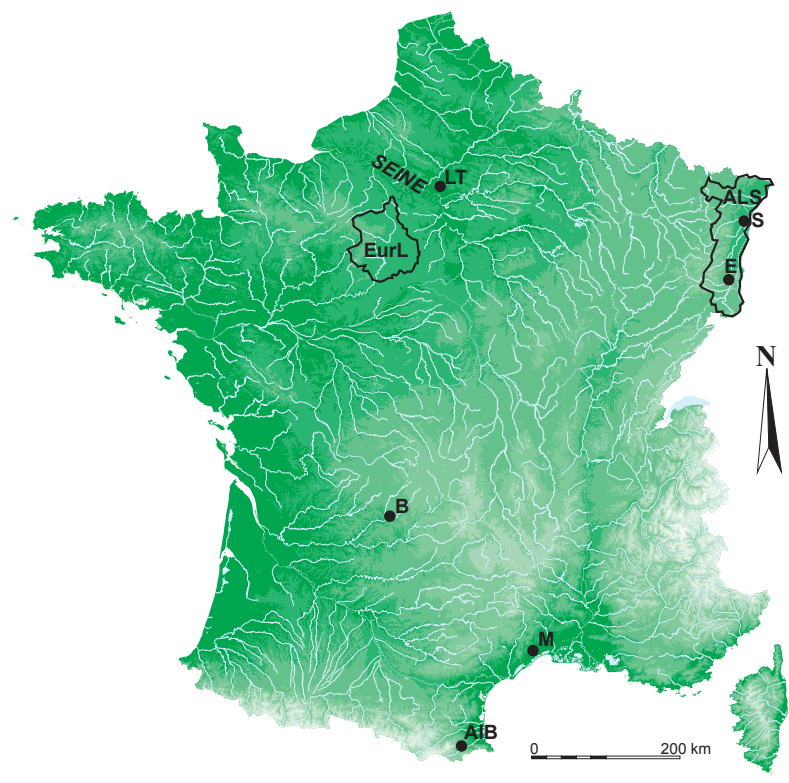

Figure 1

Localisation des principaux sites présentés dans le texte. Eurl : Eure-et-Loir ; Als : Alsace ; B : Brive ; LT : Le Thillay ; $\mathrm{E}$ : Ensisheim ; $\mathrm{M}$ : Montpellier ; S : Strasbourg ; Alb : Amélieles-Bains (modifié d'après Open Street Map).

géomorphologique et pédosédimentaire multi-scalaire et ouvertures mécaniques systématiques pour aider à trouver les sites archéologiques menacés de destruction, mais aussi pour reconstituer les cadres de vie des sociétés du passé et penser les changements environnementaux et sociaux. Il propose un survol d'ensemble, non exhaustif, du Paléolithique à l'époque contemporaine, dans des contextes géologiques et géomorphologiques différents : le bassin de Paris, le Sud-Ouest, le Languedoc et l'Alsace (fig. 1). Le choix de ces différents cas vise à montrer que la démarche ne s'applique pas seulement au Paléolithique et au Mésolithique, qu'elle ne se limite pas à un nombre restreint de contextes géographiques, qu'elle est commune à la plupart des régions françaises et qu'elle est développée par l'ensemble des opérateurs publics en charge des diagnostics archéologiques.

[1] HUREL \& COYE 2011.

[2] BRAVARD et al. 1989.

[3] LeBret \& HALBout 1991, Krier 1994, PAStRe 1994.

[4] Mordant 1994, Auxiette \& Dubouloz 2009.

[5] MaRmet et al. 2002, Dubouloz 2003, DAugas \& Bonin 2006, MALRAIn 2008, DePAEPE \& SÉARA 2010 , GUILHOT 2018. 


\section{LE TRAVAIL EN AMONT}

\section{PRÉPARER LES OPÉRATIONS DE DIAGNOSTIC}

Le principe d'étude géologique préliminaire a été formalisé notamment lors des travaux archéologiques préalables à l'autoroute $A 5$, dans l'est de la France, entre Melun et Chaumont. Le dépouillement des cartes géologiques, pédologiques, topographiques et de la littérature existante, combiné au suivi des sondages géotechniques a ainsi permis de définir : 1/ l'épaisseur des niveaux susceptibles de livrer des vestiges archéologiques ; $2 /$ les unités lithostratigraphiques régionales quaternaires ; 3 / le cadre chronostratigraphique ; 4/ les zones favorables aux études paléo-environnementales [6]. Cette démarche constitue une des raisons de la réussite de cette opération.

De fait, une lecture critique des cartes géologiques apparaît d'un grand intérêt pour identifier les contextes favorables à la conservation de sites archéologiques. Ces documents sont toutefois hétérogènes et de qualité inégale en fonction des spécialités et de l'intérêt des différents auteurs. En Ile-de-France, les formations loessiques pléistocènes ont ainsi été regroupées, quelle que soit leur nature, comme des «limons de plateaux ». Surtout, l'épaisseur minimale à partir de laquelle ils ont été cartographiés varie d'une carte à l'autre, de trente centimètres [7] à plus de trois mètres [8]. S'il existe de très rares cartes géomorphologiques [9], des recherches géomorphologiques ont été financées par des collectivités territoriales dès les années 1980 pour identifier les sites archéologiques menacés par les travaux d'aménagement du territoire [10]. Les travaux universitaires récents menés sur des tronçons de vallées [11] apparaissent également fondamentaux. Pour préciser ces premières approches et pour identifier les formations superficielles peu étendues, l'examen des cartes topographiques, complété par les données Lidar quand elles existent, est également d'un grand intérêt. Si, par le passé, les expériences de prospection préalable à l'implantation des sondages mécaniques ont montré tout leur intérêt [12], cette méthode est aujourd'hui trop rarement employée.

[6] KRIER 1994.

[7] POMEROL et al. 1969.

[8] MÉGNIEN 1987.

[9] CLOOTS et al. 1973.

[10] Lebret \& Halbout 1991, Krier 2004.
De façon générale, une phase d'étude géologique et géoarchéologique préparatoire est loin d'être systématique. Son inscription dans les prescriptions de diagnostic par les services régionaux de l'archéologie constituerait un levier important pour généraliser la démarche.

\section{DES PROGRAMMES CIBLÉS DE RECHERCHES POUR CERTAINES PÉRIODES OU CATÉGORIES DE SITES ARCHÉOLOGIQUES}

En Ile-de-France, la démarche initiée à partir de 2007 par Frédéric Blaser, dans le cadre du programme d'activité scientifique de I'INRAP consacré au Paléolithique et au Mésolithique de la région [13], a permis la découverte de plus d'une trentaine de nouveaux sites paléolithiques, a fait l'objet d'une tableronde en cours de publication [14] et a inspiré des programmes similaires en Eure-et-Loir et en Alsace.

En effet, depuis 2013 en Eure-et-Loir, un programme de collecte systématique des données relatives aux formations limoneuses d'origine éolienne est piloté par le conseil départemental, en collaboration avec les services de l'État, I'INRAP et l'agglomération de Chartres. L'objectif de ce programme de recherche est d'analyser les caractéristiques spatiales de la couverture limoneuse dans cette zone située en limite sud de la zone de couverture continue à subcontinue des lœss, à plus de $150 \mathrm{~km}$ des sources de limons (Manche). La documentation concernant les enregistrements pédosédimentaires est recensée et harmonisée à l'aide d'un système d'informations géographiques, des prospections de terrain, des diagnostics avec des sondages profonds tous les $50 \mathrm{~m}$ jusqu'au substrat tertiaire et des fouilles archéologiques. Les séquences présentes sont analysées, ainsi que leur variabilité à l'échelle du département et leur intégration dans les modèles chrono-stratigraphiques du nord de la France et de l'Europe occidentale. Les résultats montrent que le potentiel pédosédimentaire du département est riche pour la fin du Quaternaire, et plus particulièrement, pour le Pléistocène supérieur et l'Holocène. Des accumulations de plus $7 \mathrm{~m}$ sont conservées localement et les caractères des séquences (texture, carbonatations, cycles climatiques observables) ont permis de délimiter six zones dans la couverture limoneuse de plateau et

[11] Par exemple CASTANet 2008.

[12] Deschodt 2010, BruXelles 2010.

[13] Souffi et al. 2009.

[14] https://www.inrap.fr/de-l-ile-de-france-l-europedu-nord-ouest-les-peuplements-humains-avantle-13938 
cinq dans les formations de versant [15] (fig. 2). Les enregistrements de I'Eémien et du Début Glaciaire weichsélien y sont bien représentés, notamment à Chaudon. Bien que les séquences de plateau soient hydromorphes et polyphasées, elles peuvent contenir des sites du Paléolithique bien préservés, comme à Illiers-Combray [16].

Engagé en 2015, le programme collectif de recherche (PCR) Paleoels, financé par le Ministère de la Culture (DRAC Grand Est), fédère des acteurs du CNRS, de I'INRAP, d'Archéologie Alsace, de l'université et du secteur privé ; il vise à reconstituer les comportements des sociétés paléolithiques et mésolithiques dans les environnements changeants d'Alsace. Pour ce faire, les premières années du projet ont été consacrées à mieux comprendre les formations sédimentaires et les sites archéologiques connus [17]. Plusieurs campagnes d'étude des coupes accessibles, notamment dans les carrières de loess encore en activité, ont ainsi été menées. Elles ont été accompagnées d'un inventaire de la littérature et des travaux universitaires existants.

[15] BORDERIE et al. 2017.

[16] BORDERIE et al. 2019.

[17] https://fr.calameo.com/books/00372503877bf49 f2aa26

[18] WUSCHER et al. 2019.
Elles ont été réalisées en parallèle d'un réexamen de la documentation et des collections archéologiques. Un aller-retour permanent entre les travaux du PCR et les opérations d'archéologie préventive ainsi qu'une confrontation des travaux archéologiques et géomorphologiques ont permis de dégager des tendances régionales sur la conservation et la localisation des vestiges. Ainsi, des sites mésolithiques et tardiglaciaires peuvent être conservés partout, sauf dans des zones localisées, généralement dans l'axe des cours d'eau, fortement érodées à partir de la Protohistoire. Des horizons contemporains du Paléolithique supérieur ancien sont fréquemment mis au jour, notamment dans les loess, lors des travaux d'aménagement du territoire. Il n'est pas encore possible de dire si le faible nombre d'indices et de sites de cette période tient aux méthodes de diagnostic employées ou à une réelle déprise humaine. Les horizons du Paléolithique moyen et inférieur localisés sur les bordures du Fossé Rhénan ne sont pas rares, notamment sur les versants, à des profondeurs très variables, ce qui explique le nombre important d'indices archéologiques de ces périodes. Les travaux du PCR se poursuivent maintenant à des échelles plus locales et le périmètre du projet a été élargi au Jura alsacien et au Massif Vosgien. Ils s'orientent également vers une réflexion sur les premiers peuplements alsaciens et sur les territoires paléolithiques et mésolithiques [18].

\section{Figure 2}

Zonage de la couverture limoneuse d'origine éolienne sur le département d'Eureet-Loir (d'après BORDERIE et al., 2017). Une variation du type de séquence peut être constatée selon un gradient globalement nord-sud avec une épaisseur moyenne plus importante au nord, où des horizons carbonatés peuvent être conservés en profondeur. Ces caractéristiques ont un impact sur la conservation des vestiges préhistoriques.

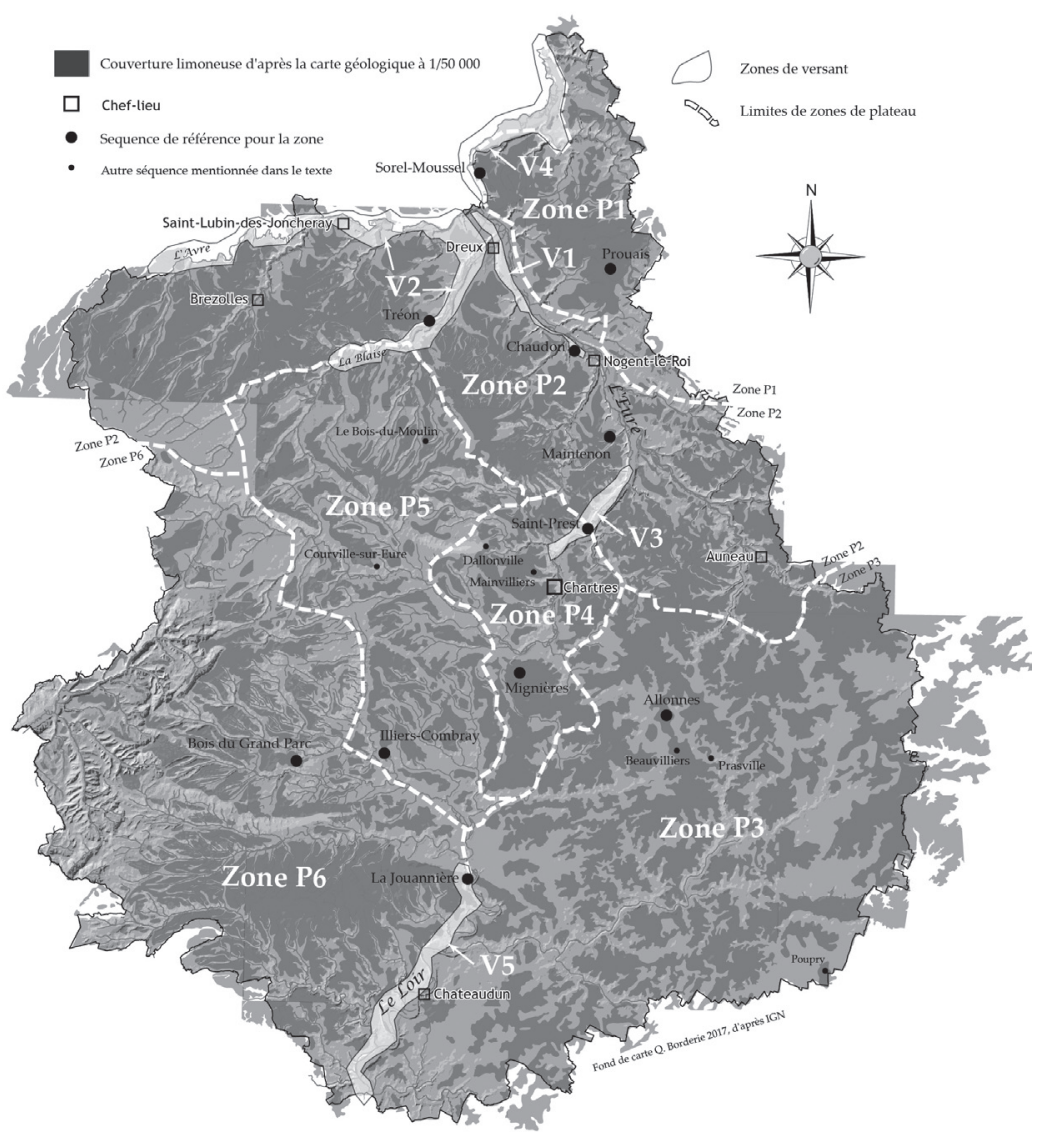


Cette démarche pourrait être étendue à la recherche de sites peu documentés d'autres périodes, comme par exemple les sites de berges ou les villages médiévaux et modernes désertés suite aux crues dévastatrices du Petit Âge Glaciaire dans la vallée du Rhin. Un inventaire critique des découvertes anciennes, des études documentaires, des prospections pédestres et subaquatiques et des sondages ciblés devraient permettre de documenter ces sites évoqués par les sources écrites. Surtout, même si les chiffres sont difficiles à évaluer précisément, moins de $25 \%$ des surfaces aménagées en France faisaient l'objet d'un diagnostic archéologique au début des années 2010 [19]. Compte-tenu de ce cadre d'intervention finalement restreint, une meilleure prise en compte des travaux géomorphologiques dans les choix des terrains qui font l'objet de prescriptions de diagnostics serait pertinente.

\section{LA DÉTECTION DES SITES}

\section{LA RECHERCHE DE LA PRÉHISTOIRE ANCIENNE}

Dans le nord de la France, l'intérêt archéologique de la couverture loessique et du système de terrasses alluviales est connu depuis le XIXe siècle [20]. Les premières opérations d'archéologie préventive ont été réalisées sur des gisements paléolithiques dans les années 1970 et se sont systématisées dans les années 1990 [21]. La couverture loessique fait l'objet de travaux géomorphologiques et d'archéologie préventive depuis plusieurs décennies, et les sondages sont généralement concentrés sur les versants exposés au nord et au nord-est, où les successions stratigraphiques renferment assez systématiquement des paléosols et des sites archéologiques [22]. Dans le sud-ouest de la France, le potentiel du sous-sol en termes de conservation de sites paléolithiques est également connu de longue date, mais les formations sédimentaires susceptibles de livrer des vestiges sont localisées et discontinues, le plus souvent dans des cavités karstiques. La localisation et le maillage des tranchées sont

[19] Collart 2012.

[20] ANTOINE et al. 2011.

[21] Ibid.

[22] LOCHT et al. 2010.

[23] Colonge et al. 2004, BruXELLES 2010.

[24] Leur matrice sédimentaire a été érodée.

[25] BRUXELLES et al. 2003.

[26] FORRER 1925.

[27] WUSCHER et al. 2013. donc plus concentrés dans ces formations. Ainsi, avant les travaux de l'aéroport de Brive, un travail de prospection archéologique et géomorphologique préalable aux ouvertures mécaniques a été mené. Par la suite, aux sondages systématiques et régulièrement espacés a été préférée une concentration des tranchées dans les dolines, de manière rayonnante, conformément à la logique des dynamiques sédimentaires, ce qui a conduit à la découverte d'un gisement attribué au Paléolithique moyen ancien [23]. Sur les terrasses de la Garonne, les couvertures limoneuses qui scellent les dépôts graveleux ont eu une histoire polycyclique. Les vestiges anciens sont souvent résidualisés [24] et incorporés dans un petit niveau de graves régulièrement confondu avec le corps de la terrasse graveleuse. L'identification de ce phénomène a conduit les équipes à systématiquement traverser ce premier niveau de graves, ce qui a permis la découverte de nombreux vestiges [25].

La moitié sud-est de l'Ile-de-France et la bordure de la plaine d'Alsace présentent des caractères communs avec le Sud-Ouest et des vestiges archéologiques paléolithiques sont susceptibles d'apparaître à des profondeurs variables, parfois faibles, «presque à fleur de terre » [26]. En revanche, la moitié nordouest de l'Ile-de-France de même que les terrasses alluviales et les collines alsaciennes sont caractérisées par des formations sédimentaires continues, similaires à celles du nord de la France. Ces formations sont donc sondées à l'aide d'un maillage régulier et discontinu de sondages profonds par une équipe mixte composée d'archéologues et de géomorphologues. En Ile-de-France et en Alsace, elles sont moins connues et doivent, dans l'état actuel des connaissances, faire l'objet d'un maillage plus systématique pour comprendre leur géométrie, avant de focaliser la prospection sur les paléosols. Le diagnostic mené durant l'hiver 2013 sur 22 ha de la commune du Thillay (95), situé sur la partie occidentale du plateau éocène de la plaine de France, en surplomb de la vallée du Crould, illustre bien cette démarche. Trente sondages ont permis de délimiter l'extension d'une séquence de limons lœssiques de trois à quatre mètres d'épaisseur contenant plusieurs paléosols (fig. 3). Trois niveaux archéologiques ont été mis en évidence [27]. Le niveau inférieur est situé à la base d'un paléosol (probablement un sol brun arctique), qui a livré une pièce bifaciale (fig. 4) et des pièces à dos. Les caractéristiques pédosédimentaires de la séquence et le type d'assemblage semblent indiquer un âge weichsélien moyen (environ entre 55000 et 30000 ans avant l'actuel). Le deuxième niveau est un paléosol de type gley de toundra qui a livré une dent d'équidé datée par la méthode du radiocarbone 

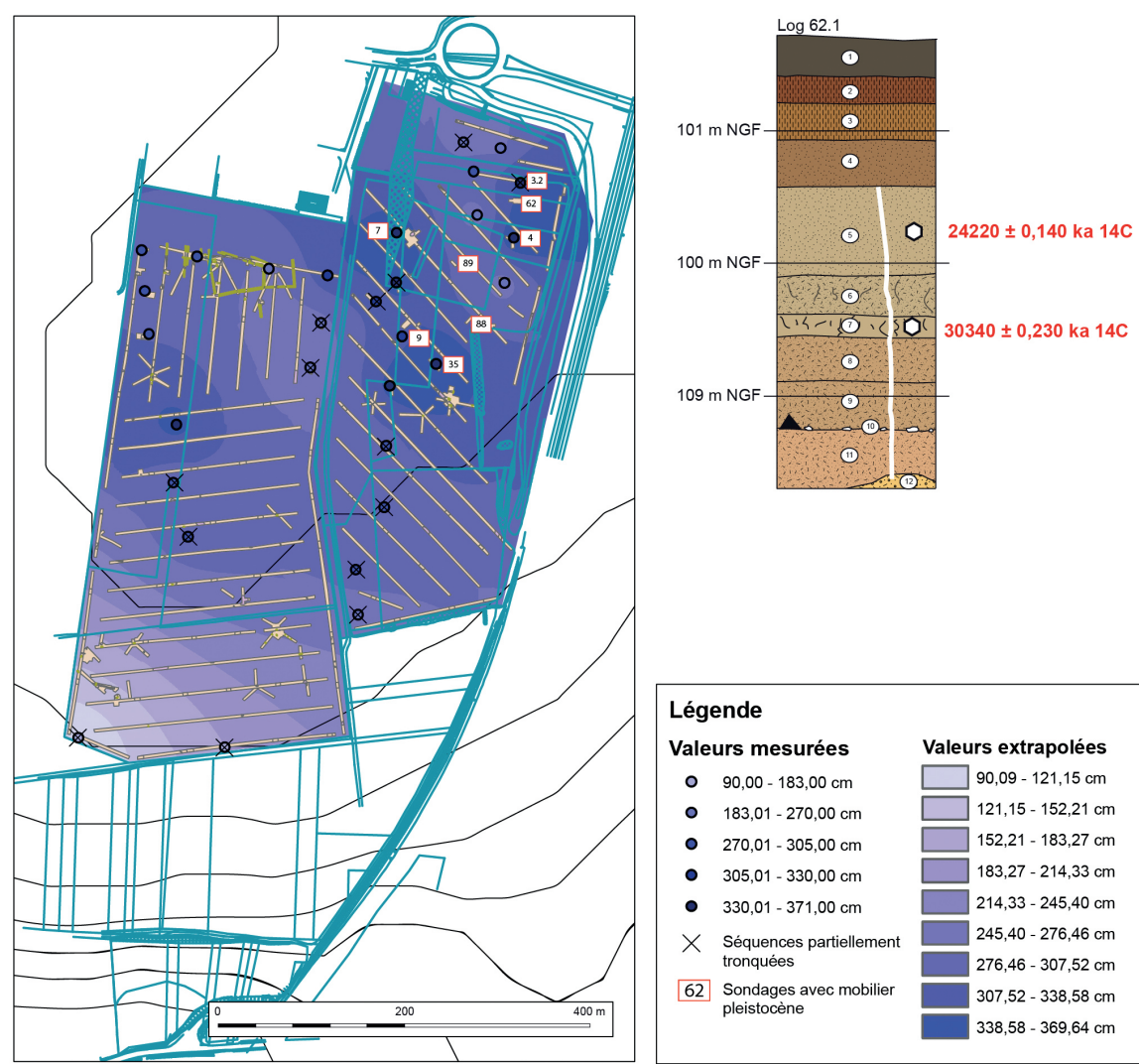

Figure 3

Le Thillay, Val-d'Oise, épaisseur des formations pléistocènes et log du sondage 62.1 représentatif de l'emprise (le triangle placé sur la coupe indique la position de la pièce bifaciale, les datations ont été réalisées à partir des restes osseux découverts dans le sondage 3.2 , voisin du sondage 62.1).
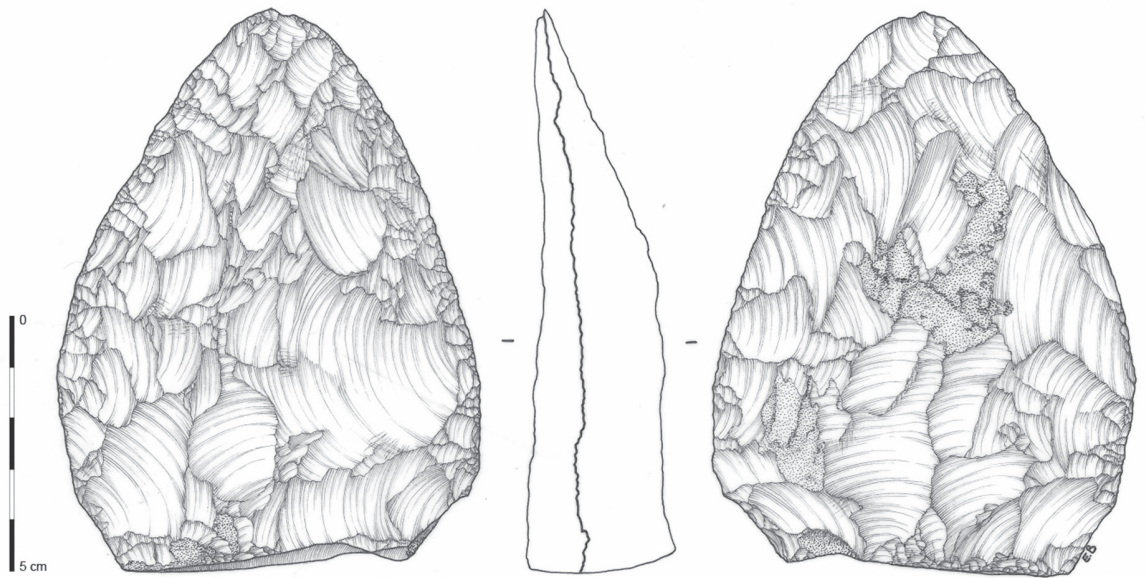

Figure 4

Le Thillay, Val-d'Oise, pièce bifaciale découverte dans l'horizon attribué au weichsélien moyen, environ entre 55000 et 30000 ans avant l'actuel (dessin E. Boitard/INRAP).

de 30,340 \pm 0,23 ka avant l'actuel [28]. Le troisième niveau est constitué de lœss calcaires dans lesquels a été découvert un fragment de phalange d'équidé brûlé qui a livré un âge radiocarbone de 24,220 \pm 0,14 ka avant l'actuel.

\section{LES INDICES DIFFUS DU MÉSOLITHIQUE}

Le Mésolithique s'inscrit dans une période tempérée caractérisée par un couvert forestier étendu. Les vestiges de la période sont parfois représentés par des fosses et des sépultures, mais le plus souvent par des concentrations de mobilier [29]. Sur la majeure partie des terrains menacés par des travaux d'aménagement du territoire, notamment les plateaux ou les versants, la rareté des dépôts sédimentaires de la période n'a pas été favorable à la conservation des gisements, même si des petits thalwegs ont pu piéger du mobilierou si des restes lithiques ont pu être enterrés par la faune du sol [30] ou piégés dans des chablis [31]. Des successions stratigraphiques de la période sont toutefois

[28] Datation financée par le Programme d'Activité Scientifique de I'INRAP « Recherches Archéologiques préventives dans le Bassin Parisien du Pleistocène à I'Holocène : Chronologie, caractérisation culturelle et fonctionnement des sites », sous la direction de Bénédicte Souffi, Boris Valentin et Fréderic Blaser.

[29] DuCROCQ 2010.

[30] WuSCHER et al. 2012.

[31] DuCROCQ 2010. 
conservées en fonds de vallées, en contexte sableux et au pied de certains versants. Mais, même dans ces contextes topographiques particuliers, les vestiges sont généralement dilatés du fait des processus pédologiques (bioturbations) qui caractérisent l'Holocène avant les défrichements néolithiques et protohistoriques [32]. Bien souvent, les vestiges du Mésolithique apparaissent donc au même niveau que les vestiges et les structures en creux des périodes plus récentes. Les spécialistes de la période insistent donc sur l'intérêt des phases d'étude documentaire, prenant notamment en compte les découvertes des prospections pédestres. Ils insistent aussi sur l'indispensable participation de spécialistes du mobilier lithique et sur la nécessaire synergie entre archéologues et géomorphologues [33]. Les travaux menés à Ensisheim dans la vallée de l'III illustrent bien cette démarche. Identifiés en 2015 [34], les horizons tardiglaciaires et holocènes font depuis l'objet de travaux réguliers (fig. 5). La plaine de l'Ill a été façonnée après l'incision et la migration du Rhin à l'est du cône de la Hardt, dont les derniers dépôts ont été datés par luminescence stimulée optiquement (OSL) de $16200 \pm 1200$ ans avant le présent [35]. Le remplissage de la plaine est constitué de sables pédogénéisés recouverts par des limons attribués au Tardiglaciaire sur la base de datations par OSL (unités 8 à 5, fig. 6). Un sol noir se développe sur ces dépôts (unité 3 , fig. 6) puis un luvisol (unité 2, fig. 6).

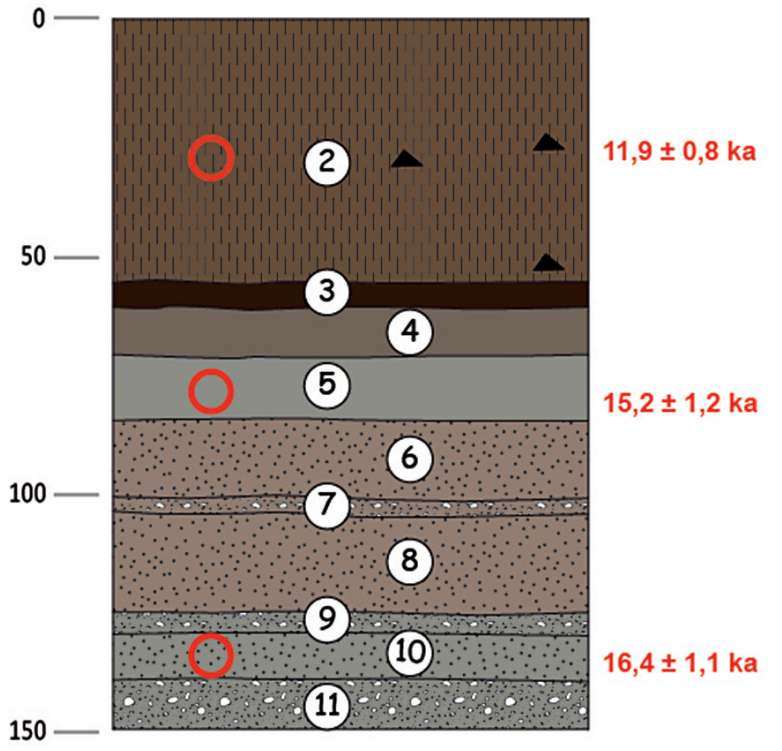

Figure 6

Succession stratigraphique de la nappe II. Les triangles indiquent la position du mobilier mésolithique. Les ronds et les chiffres rouges indiquent les âges OSL. Les deux âges supérieurs sont légèrement trop vieux, ce qui tient aux limites de la méthode.

[32] Ibid., Confalonieri \& Le Jeune 2012.

[33] DUCROCQ 2010.

[34] PieRrevelcin et al. 2016.

[35] WUSCHER et al. 2018.

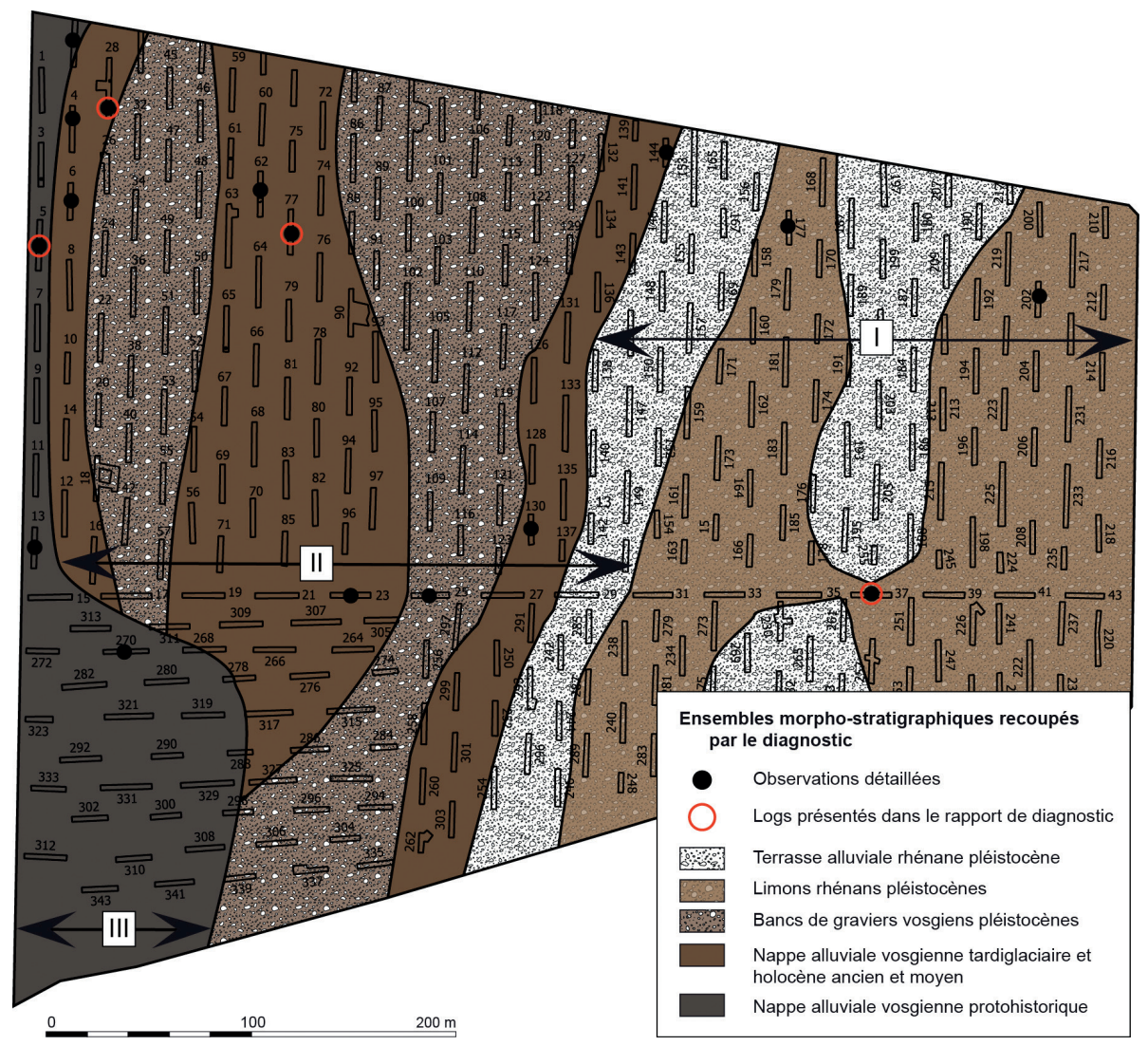

Figure 5

Cartographie des trois nappes alluviales de la plaine de l'Ill à Ensisheim dans l'emprise du diagnostic mené durant l'hiver 2018-2019:

I : nappe weichsélienne ; II : nappe tardiglaciaire et holocène ;

III : nappe protohistorique. 
Localement, cette succession stratigraphique est tronquée par des dépôts torrentiels contenant des fragments de céramiques protohistoriques [36]. Au sein de ces niveaux, un suivi géomorphologique régulier, 2 à 3 demi-journées par semaine, a permis de cartographier les dépôts, de délimiter les secteurs tronqués à partir de la Protohistoire et de cibler les paléosols, révélant des vestiges du Mésolithique moyen, récent et final [37]. Cette succession stratigraphique peut se suivre de Mulhouse à Colmar [38] et elle a livré une pièce mésolithique à Oberhergheim [39].

\section{DE LA PRÉHISTOIRE RÉCENTE AUX PÉRIODES HISTORIQUES DANS LES SECTEURS À FORT RECOUVREMENT SÉDIMENTAIRE}

À la fin de la dernière période glaciaire, il y a 20000 ans, le niveau général des océans, 120 à 130 m plus bas qu'aujourd'hui, conduit les fleuves à creuser de profonds canyons pour rejoindre le niveau de base. À partir du réchauffement climatique post-glaciaire (après 18000 avant l'actuel), les calottes glaciaires fondent et le niveau des mers remonte très rapidement jusqu'au début de l'Holocène, puis plus progressivement. Ainsi, à la fin du Mésolithique, il y a 8000 ans, le niveau marin est encore entre 7 et $9 \mathrm{~m}$ sous l'actuel [40]. Les fleuves accompagnent cette remontée marine en comblant leurs anciennes rias. Le Lez, petit fleuve côtier qui traverse la ville de Montpellier et se jette dans la Méditerranée, en est un bon exemple. De nombreuses opérations d'archéologie préventive ont été menées dans sa plaine littorale depuis plus de 20 ans et les modes opératoires ont ainsi été adaptés à ce contexte particulier. L'opération de diagnostic menée en 2017 dans le cadre de l'extension de la station d'épuration de Montpellier, à Lattes, en est emblématique (fig. 7). Profond de plus de 9,00 m, un des sondages effectués a permis la mise au jour des occupations qui se sont succédées dans la plaine alluviale au cours des huit derniers millénaires (fig. 8). Dans le même temps, un travail a été mené sur les changements dans la dynamique du Lez afin de mieux appréhender les métamorphoses fluviales en relation avec la remontée marine et les rythmes du peuplement dans cette zone en mutation constante. Quatre grandes phases d'évolution des milieux sont identifiées, qui font écho aux données déjà acquises autour de Lattes [41]. La phase a, datée au moins de la fin du Mésolithique, correspond à un paysage de plaine d'inondation humide, à tendance marécageuse. Pour cette période, on n'a trouvé que peu de vestiges archéologiques, mais l'on sait que la plaine est occupée comme en témoignent les

[36] Pierrevelcin et al. 2016, Roth-Zehner et al. 2016.

[37] Roth-Zehner et al. 2016, Roth-Zehner et al. 2019.

[38] BlondeAu \& Wuscher 2017, BlondeAu 2018.

[39] BLONDEAU 2018.

[40] VACCHI et al. 2016.

[41] Daveau 2007, Marsac et al. 2019.

\section{Figure 7}

Sondage de diagnostic préalable à l'extension de la station d'épuration de Montpellier, à Lattes.

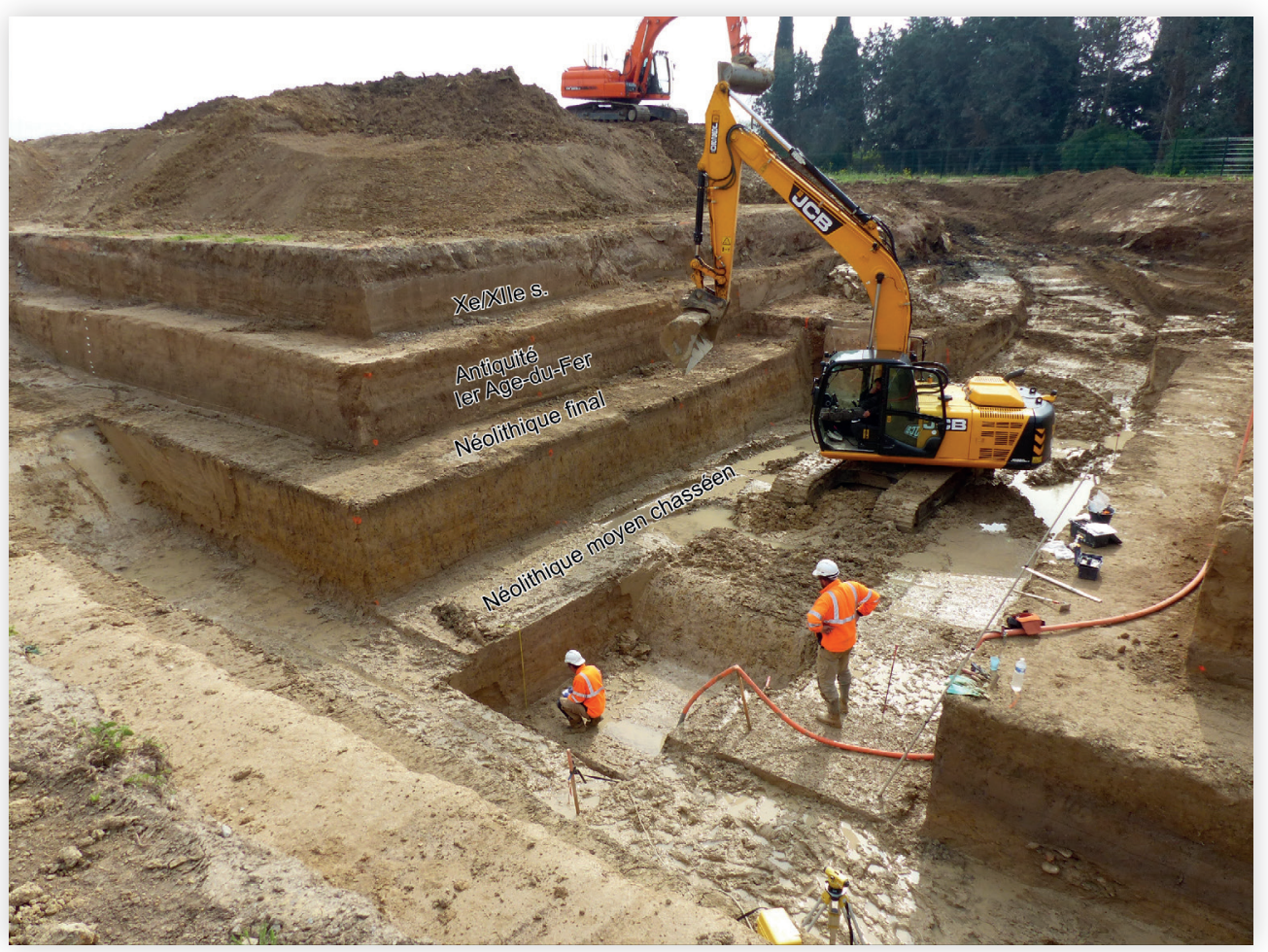




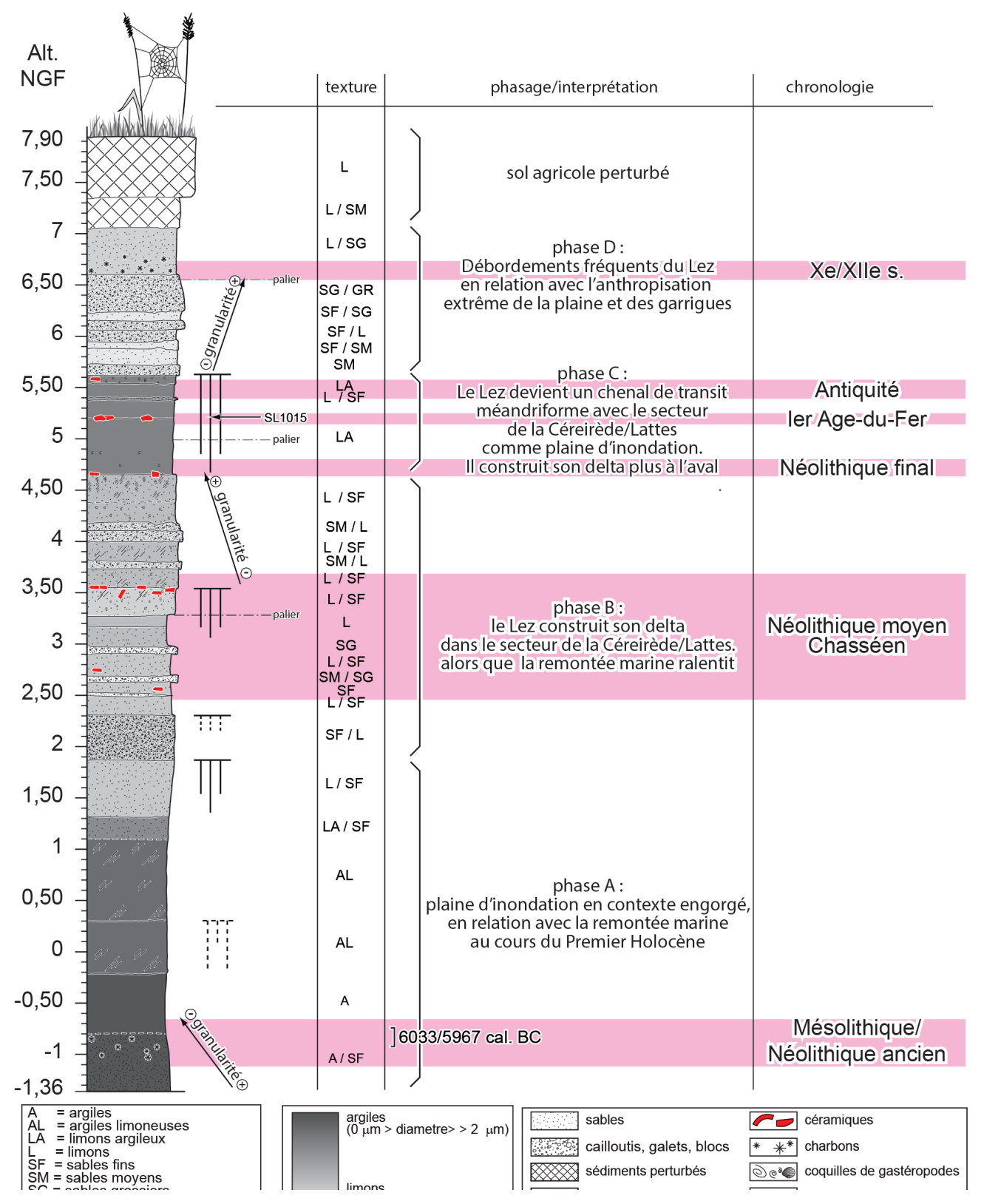

Figure 8

Stratigraphie type de la basse plaine du Lez depuis le Néolithique ancien : l'exemple de la station d'épuration de Montpellier, à Lattes (Hérault).

quelques découvertes ponctuelles (essentiellement des fosses) ou les niveaux charbonneux mis au jour dans plusieurs sondages. La phase $b$, datable du Néolithique moyen, montre un cours d'eau très énergique, avec des chenaux larges et partiellement tressés, associé à de nombreuses défluviations. Les sédiments qu'il dépose sont systématiquement grossiers, sableux à limono-sableux. Pour cette période, les vestiges sont bien plus nombreux et correspondent à des occupations d'ampleur comme à Port-Ariane [42], à des installations plus ponctuelles comme le tumulus des Rauzes Basses [43] ou des fosses et des silos et des traces d'habitat, comme sur le site de La Céreirède fouillé en 2003 [44]. La phase $c$ inaugure un changement fondamental dans le fonctionnement du fleuve, qui va perdurer jusqu'à aujourd'hui. Évoluant à partir d'environ 2500 ans avant notre ère vers un cours d'eau à chenal unique, sa plaine d'inondation n'est plus exhaussée que par des limons et argiles de débordement. Dans ce nouveau cadre paysager, I'occupation de la plaine s'intensifie et nombreuses en sont les traces à partir du Néolithique [45]. La phase d, d'âge historique, est marquée par un fonctionnement plus dynamique du fleuve avec de fréquents débordements qui sont mis en relation avec la forte anthropisation de l'ensemble du bassin versant.

Dans les vallées des zones montagnardes et semi-montagnardes, les formations tardiglaciaires et holocènes sont discontinues et très localisées. Ces

[42] DaVeau 2007.

[43] MARSAC et al. 2019.

[44] JUNG et al. 2017.

[45] Daveau 2007, OtT \& Tarrou 2013, Rascalou \& BeL 2013, RAUX et al. 2013. 
secteurs, caractérisés par des dépôts grossiers, se prêtent mal aux carottages et leur observation n'est possible qu'à partir des affleurements mis au jour par les torrents, dont les rives sont souvent défendues par des murs ou des empierrements qui n'ont fait l'objet que de peu d'études. Il est donc difficile de prédire la sensibilité archéologique de ces milieux sans des ouvertures mécaniques. Une lecture conjointe, géomorphologique et archéologique, et une adaptation du maillage des tranchées au sous-sol sont donc indispensables lors des diagnostics. Celui qui a été mené à Amélie-les-Bains, dans la moyenne vallée du Tech (Pyrénées-Orientales, fig. 9), permet d'illustrer la démarche [46]. Il est localisé sur une bande de 1 $\mathrm{km}$ de long et de 0,3 km de large délimitée au nord par un versant très raide façonné dans les micaschistes du Canigou et les calcaires du Mésozoïque (synclinal d'Amélie-les-Bains) dans lequel s'ouvrent trois bassins-versants torrentiels. Au sud, il domine d'une dizaine de mètres le lit actuel du Tech, fleuve dont le régime hydrologique combine des caractères méditerranéens et montagnards, et qui est marqué par des crues violentes et paroxysmiques, dont la plus célèbre, l'aiguat de 1940, est encore très présente dans les esprits. Une première série de tranchées a été déployée sur l'ensemble de l'emprise, ce qui a permis de cartographier le sous-sol et d'identifier une accumulation de colluvions fossilisant plusieurs paléosols, une berge antique et médiévale ainsi que deux grands cônes de graviers de l'époque moderne. Dans un second temps, les tranchées ont été concentrées sur la berge et les paléosols. Sur la berge, cela a permis de

[46] Pezin \& Wuscher 2005.

[47] Ibid.

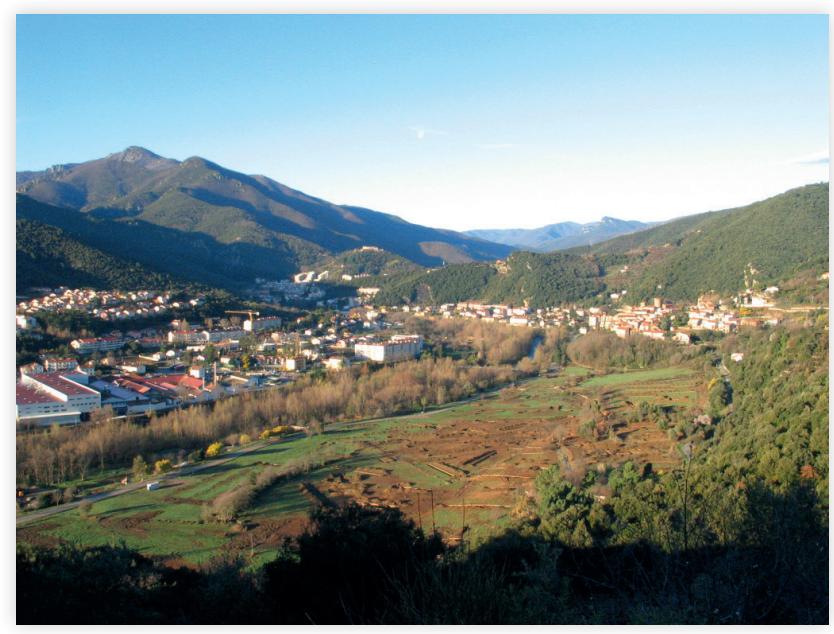

Figure 9

Amélie-les-Bains, vue de l'environnement du diagnostic archéologique (cliché : Annie Pezin/INRAP).

mettre en évidence des murs, un sol construit de type calade et des fosses constitutifs d'un établissement rural médiéval. Dans les paléosols, les tranchées ont mis au jour un bâtiment sur poteaux de $25 \mathrm{~m}$ de long pour $7 \mathrm{~m}$ de large, avec des murs de terre massive conservés sur 10 à $20 \mathrm{~cm}$ de hauteur, plusieurs foyers, ainsi qu'un abondant mobilier et de nombreuses céramiques écrasées en place, attribuées au Bronze final IIIb (environ 900-800 avant notre ère). Dans le même secteur, a été découvert un ensemble de l'Antiquité Tardive. Structuré autour d'un chemin, il comprenait un bâtiment, une forge, des fosses de plantation de vignes, des murs de terrasse et un ensemble de sépultures dont les emplacements étaient signalés par des amas de pierres [47]. La fouille de cet ensemble funéraire a mis au jour trois tertres funéraires construits en pierres d'environ $5 \mathrm{~m}$ de diamètre et de plus de $1 \mathrm{~m}$ de haut (fig. 10). Fossilisés par environ $1 \mathrm{~m}$ de

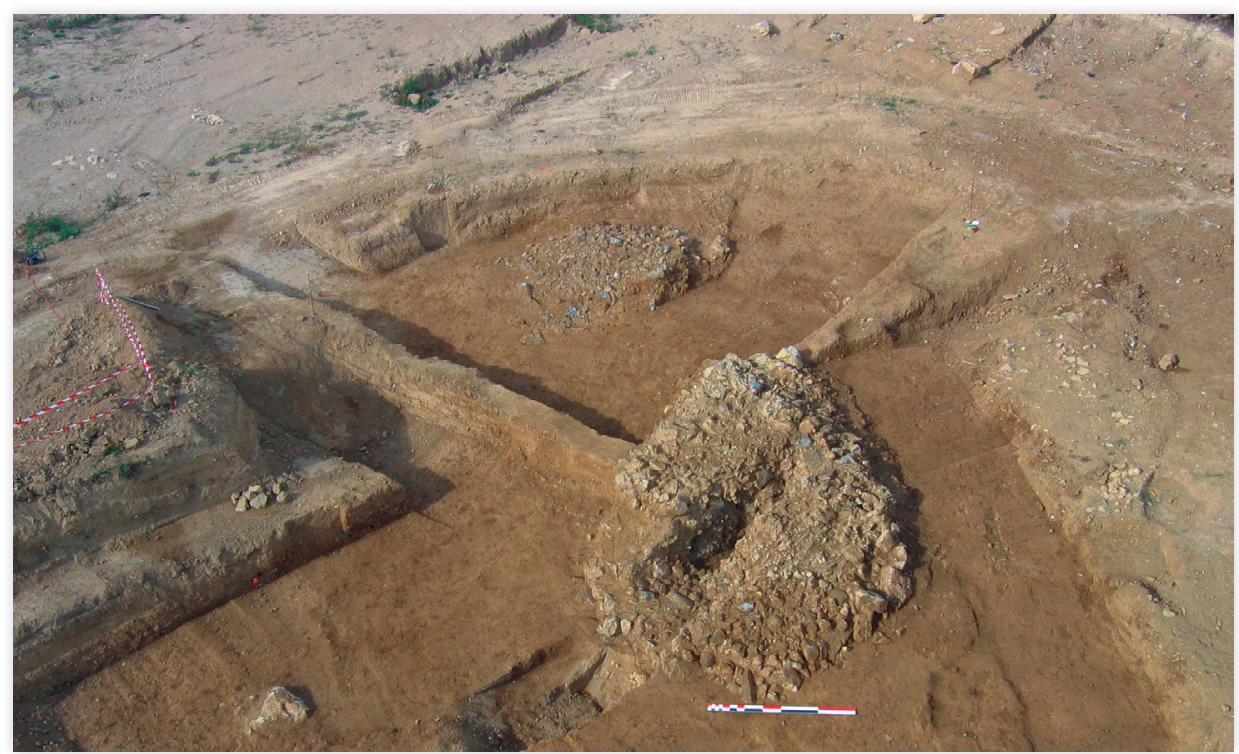

Figure 10

Amélie-les-Bains, vues des tertres funéraires du Bronze final (cliché : Patrice Wuscher). 
colluvions, ils émergeaient entre les tombes antiques et ont été pris pour des structures antiques lors du diagnostic. Malgré les moyens supplémentaires alloués à la fouille, seul l'un d'entre eux a pu être fouillé et a été daté du Bronze final II, avec des réaménagements au Bronze final IIIb [48], ce qui illustre les limites du diagnostic archéologique, qui malgré une lecture pluridisciplinaire, ne constitue qu'un échantillonnage spatial limité.

Au final, l'ouverture de sondages ciblés par un géomorphologue et un archéologue peut s'avérer opportune pour identifier les formations superficielles des terrains prospectés et pour adapter au mieux la localisation des sondages, en privilégiant les paléosols et les berges. Les successions stratigraphiques sont ensuite relevées par les archéologues dans l'ensemble des sondages, tandis que les géomorphologues décrivent toutes les unités stratigraphiques à partir de séquences de référence et donnent un cadre morphosédimentaire général. L'investissement consenti pour reconstituer le fonctionnement hydrosédimentaire et géomorphologique des cours d'eau et des versants permet quant à lui de donner un cadre précis à l'évolution des paysages et de mieux comprendre les fortes interactions entre les dynamiques environnementales et les modalités de peuplement. Il convient également de prendre en compte les impacts anthropiques récents, comme celui non négligeable lié à la mécanisation de l'agriculture qui a abouti à un lissage généralisé des paysages, notamment dans les plaines, enfouissant parfois les vestiges archéologiques sous plusieurs mètres de colluvions en bas des versants.

\section{DU PATRIMOINE À LA RECHERCHE}

\section{LA GENÈSE DES FORMES DU RELIEF}

À Amélie-les-Bains, les opérations d'archéologie préventive ont permis d'écrire l'histoire géomorphologique holocène d'un tronçon de vallée qui n'était pas accessible sans terrassements mécaniques [49]. Ces opérations permettent également d'aborder des questions géomorphologiques à des échelles vastes, tant chronologiques que spatiales [50]. Ainsi, le fonctionnement quaternaire des terrasses de l'Yonne a été révélé par des travaux d'archéologie préventive [51], tandis que l'étude du remplissage des fonds de vallée du bassin français de I'Escaut permet d'aborder la néotectonique pléistocène du bassin de Paris et du bassin belgo-néerlandais et participe à reconstituer l'évolution paléogéographique de la mer du Nord [52]. En Alsace, les grandes lignes de l'évolution géomorphologique depuis la formation du Fossé rhénan ont été analysées dans les années 1980 à partir d'une synthèse des données lithostratigraphiques et topographiques disponibles [53]. Le rôle prépondérant de la néotectonique dans cette évolution a été mis en avant. L'épaisseur des formations quaternaires, notamment les plus récentes liées au dernier cycle interglaciaire-glaciaire, Eémien et Weichsélien (environ entre 130000 et 13500 ans avant l'actuel) et à l'Holocène (depuis environ 13500 avant le présent), était toutefois complexe à apprécier. Du coup, il est difficile d'évaluer la part de la néotectonique dans l'évolution géomorphologique de la moitié sud du Fossé rhénan durant les cent derniers millénaires. Si la relecture récente des nombreux forages et profils sismiques réalisés dans la région permet de mieux cerner la géométrie du fossé et de son remplissage, elle n'a pas permis d'aller beaucoup plus loin dans l'évaluation de l'épaisseur des formations sédimentaires weichséliennes [54]. Cela tient surtout à l'absence d'un horizon repère interglaciaire au sein des masses de graviers du sud de la région et au manque de nouveaux forages et de datations absolues dans les niveaux argileux et organiques intercalés au nord, autour de Strasbourg. Les diagnostics archéologiques menés préalablement à la construction de la Ligne à Grande Vitesse (LGV) Strasbourg-Paris, ainsi que ceux liés aux travaux du Contournement Ouest de Strasbourg (COS), apportent des éléments importants à ces questions. Ils ont ainsi permis de vérifier l'étendue des alluvions pléistocènes à l'ouest d'Achenheim et de préciser l'extension des loess autour de Strasbourg [55] ainsi que dans le champ de fractures de Saverne [56]. Ils permettent également de préciser l'épaisseur de certaines formations superficielles. Ainsi, l'épaisseur des loess du Kochersberg n'excède-t-elle pas en moyenne 5 à 10 mètres sur le tracé du COS, sauf sur les versants de Vendenheim et de Kolbsheim où elle atteint près de 20 mètres [57].
[48] PeZin 2014.

[49] Wuscher \& Pezin 2010.

[50] BRUXELLES 2012.

[51] CHAUSSÉ 2003.

[52] DesChODT 2014.
[53] VOGT 1992.

[54] ELSASS et al. 2006 et http://rgf.brgm.fr/page/ vosges-fosse-rhenan-pilote-rgf

[55] SCHNEIKERT 2017, MOINE et al. 2017.

[56] SCHNEIDER \& ERTLEN 2017.

[57] SCHNEIKERT 2017, MOINE et al. 2017. 
L'apport géologique et géomorphologique des diagnostics archéologiques ne se limite toutefois pas aux grands travaux. Ainsi « si certaines observations peuvent être considérées comme mineures, aucune n'est dénuée d'importance » [58]. Par exemple, le diagnostic mené sur 2,6 ha à Eckwersheim (Alsace), préalablement à la construction d'un lotissement, a mis au jour des formations oligocènes presque affleurantes [59], alors que la carte géologique et les tranchées proches du COS semblaient montrer que les collines du secteur étaient façonnées dans une épaisse couverture loessique. Au débouché de la vallée de la Thur, un diagnostic mené sur 2 ha à Pulversheim, a quant à lui mis en évidence des formations torrentielles graveleuses protohistoriques [60] dans un secteur où les dépôts cartographiés attribués au Weichsélien ont une morphologie faiblement différenciée. Surtout, l'absence de déformations reconnues dans l'ensemble des diagnostics archéologiques menés en Alsace durant les deux dernières décennies incite à relativiser la part de la néotectonique dans l'évolution du Fossé rhénan durant les derniers millénaires.

\section{LES DYNAMIQUES PAYSAGÈRES DES DERNIERS MILLÉNAIRES}

De fait, même si les diagnostics archéologiques sont implantés principalement dans les zones soumises à des aménagements lourds (autoroutes, zones d'activités et lotissements) ou autour des sites archéologiques connus, et si moins de $25 \%$ des surfaces aménagées en France font l'objet d'un diagnostic archéologique [61], la masse de données collectées pendant près de trois décennies a permis de reconstituer les milieux et les territoires des sociétés du passé, comme par exemple pour les sociétés du Paléolithique dans le Sud-Ouest [62].

Les possibilités géoarchéologiques offertes par les données de l'archéologie préventive peuvent aussi être illustrées par les travaux menés sur la plaine littorale autour de Montpellier depuis le Mésolithique. Les nombreux diagnostics et fouilles ont apporté de multiples éléments à la compréhension de leur évolution paléogéographique. En outre, ces paysages deltaïques qui sont de riches milieux vivriers (eau douce, terres cultivables, abondance des ressources ), ont été fortement investis par les populations. Réussir à dessiner ces paléopaysages devenait alors une nécessité pour mieux appréhender les modes de peuplement au cours de l'Holocène. On a ainsi choisi d'utiliser l'ensemble de la documentation disponible pour cartographier le littoral du Languedoc à différentes périodes, que ce soient les données publiées par les chercheurs travaillant sur les paléolittoraux ou sur la variation du niveau marin postglacaire, les données de fouilles, également les nombreux sondages géotechniques référencés sur le Sig en ligne Infoterre [63]. Les cartes proposées ici (fig. 11) correspondent à cette compilation de données, mais ne sont qu'un état des lieux temporaire. En effet, de nombreuses questions demeurent, en particulier sur la hauteur réelle du niveau marin selon les périodes avec des incertitudes altitudinales pouvant atteindre plusieurs mètres [64]. Pour autant, elles permettent d'appréhender les paléopaysages des différentes phases de peuplement reconnus sur le littoral et d'envisager des liens sérieux entre les populations anciennes et le territoire dont elles disposaient et qu'elles géraient en fonction de leurs besoins.

\section{DES TRACES DES PREMIERS AGRICULTEURS À L'ANTHROPOCÈNE}

Discuter la part de l'Homme et du climat dans les changements environnementaux holocènes enregistrés dans les fonds de vallées est une question ancienne en géoarchéologie. Les travaux pionniers menés en Limousin, notamment sur les chantiers de l'autoroute A20, illustrent bien cette démarche. Ils ont notamment montré l'opposition forte entre l'Holocène ancien et moyen, où l'anthropisation reste discrète et localisée, et l'Holocène récent, caractérisé par une modification majeure et irrémédiable des milieux [65]. Des travaux similaires ont été également menés en Alsace, sur les colluvions et les paléosols recoupés par la LGV Strasbourg-Paris dans les paysages loessiques du Kochersberg, avec des sols attractifs pour les agriculteurs et des matériaux sensibles à la pédogenèse et à l'érosion [66].

Dans le Bassin parisien, plusieurs centaines de sondages à la tarière et plusieurs dizaines d'opérations d'archéologie préventive menés pendant près de 30 ans ont permis de détailler l'évolution tardiglaciaire et holocène des grandes et des moyennes vallées, notamment l'Oise, la Marne, la Seine, le Crould et la Beuveronne [67]. Ils ont mis en évidence des remplissages emboîtés,

[58] DeSCHODT 2014 p. 555.

[59] CROUTSCH 2019.

[60] SCHNEIKERT \& WUSCHER 2016.

[61] COLLART 2012.

[62] BRUXELLEs \& JARRY 2011, BeRTRAN et al. 2013.

[63] http://infoterre.brgm.fr/

[64] RAYNAL et al. 2010, VACCHI et al. 2016.

[65] AlLÉE et al. 1997, AlLÉE et al. 2006.

[66] ERTLEN et al. 2012, SCHNEIDER et al. 2014.

[67] PASTRE et al. 2014. 


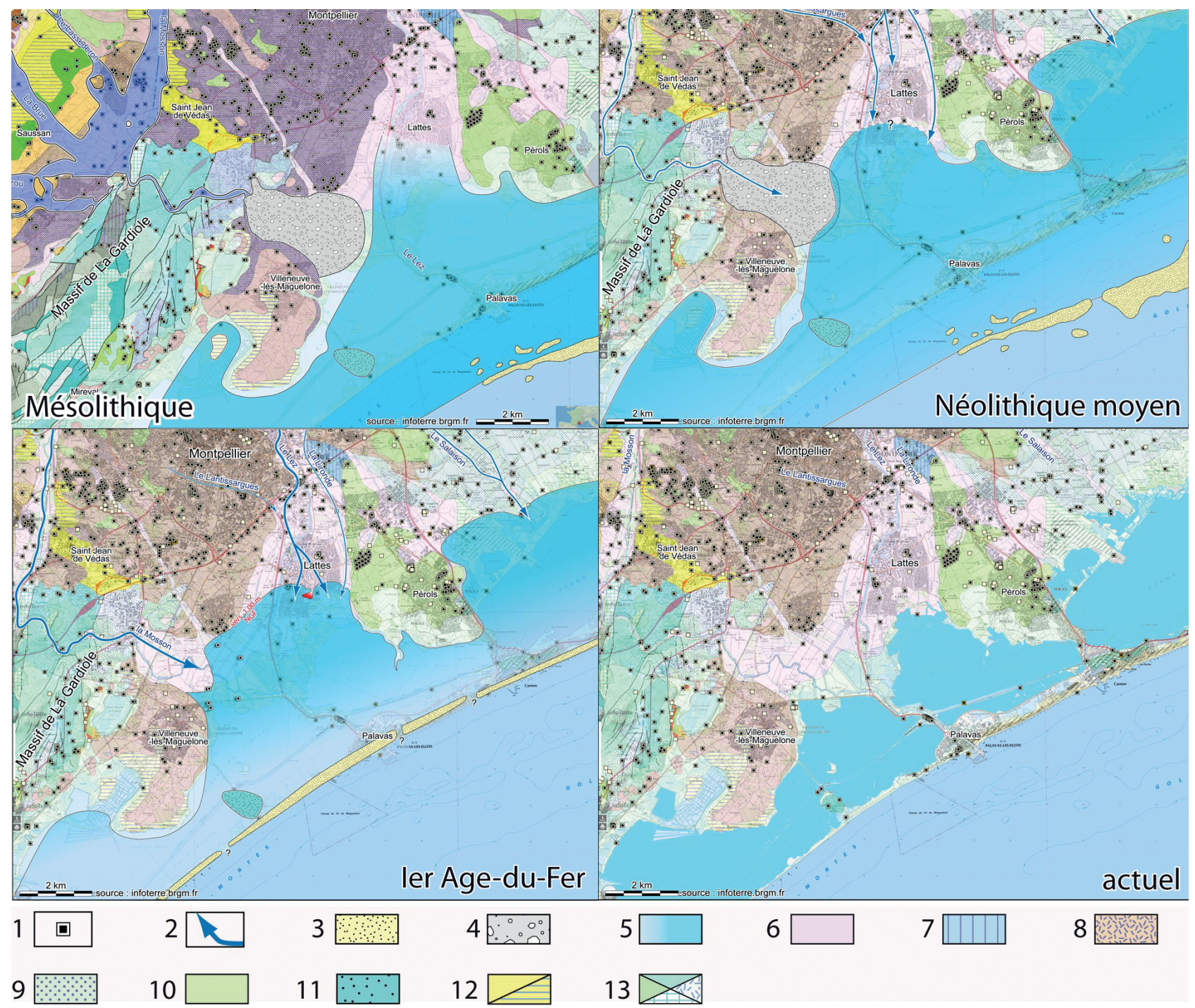

1. sondages BDSS du BRGM (http://infoterre.brgm.fr/), 2. paléochenaux du Lez, 3. ancien cordon littoral (-8,00/$10,00 \mathrm{~m}$ NGF), 4. alluvions de la Mosson au dessous des $-4,00 /-7,50 \mathrm{~m} \mathrm{NGF,} 5$. étendue schématique possible de la lagune, 6. Alluvions récentes du Quaternaire, 7. Limons et loess du Lez, 8. Colluvions anciennes du Quaternaire, 9. Faciès astien du Pléistocène, 10. Cailloutis d'origine rhodanienne d'âge villafranchien, 11. sables et grès volcano-détritiques, 12. Calcaires coquillers et marnes pliocènes, 13. Calcaires et marnes jurassiques

\section{Figure 11}

Évolution du littoral du Languedoc autour de Montpellier au cours de l'Holocène.

caractérisés par une bipartition des enregistrements, avec des dépôts principalement organiques et localisés jusqu'au Subboréal, puis des remblaiements massifs presque exclusivement minéraux. Toutefois, seules les dynamiques des grands bassins versants étaient reconstituées, ce qui n'était pas toujours compatible avec les problématiques territorialisées des archéologues [68]. Il a donc semblé intéressant de travailler sur les colluvions de quatre vallons secs drainant des petits bassins-versants lœssiques du nord-ouest de I'Ile-de-France [69]. Comme dans les grandes vallées, leur remplissage est bipartite, constitué d'un luvisol cumulique recouvert par des colluvions à dominante minérale beige, entrecoupées parfois d'horizons brunifiés d'une quarantaine de centimètres d'épaisseur. Si les luvisols présents à la base de ces séquences présentent des traces d'occupations néolithiques (charbons, fragments de céramiques, traces de rubéfaction), leur fossilisation par des dépôts massifs de colluvions est toujours d'âge protohistorique (fig. 12).

\section{[68] WuSCHER et al. 2012}

[69]_https://www.researchgate.net/publication/28558 8081 Des limons loessiques des hommes et de_I'erosion_dans_le_nord-ouest_de_I'Ile-de-France_ durant I'Holocene stratigraphie datations et micromorphologie_a_Guitrancourt_Yvelines 


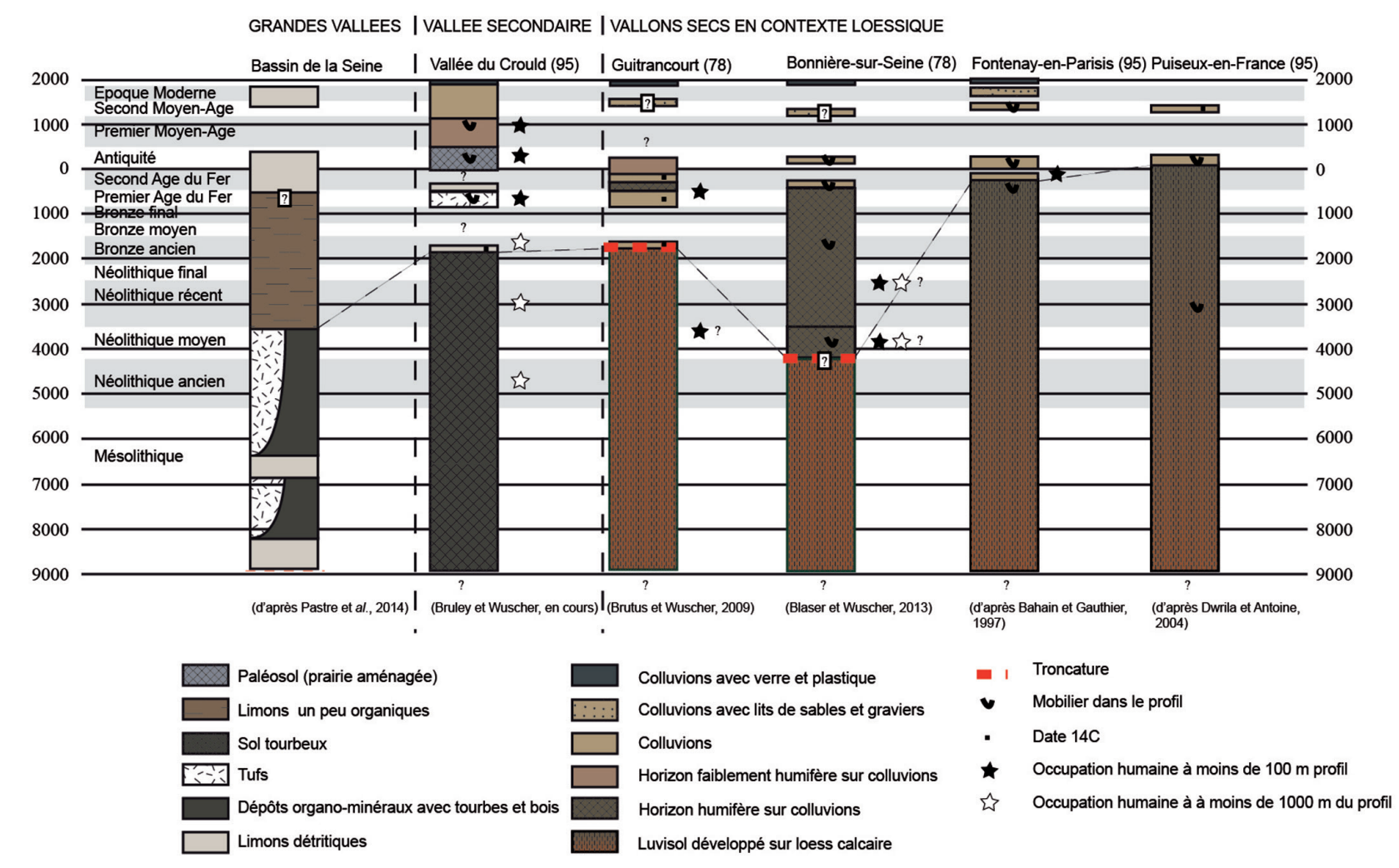

Figure 12

Proposition de corrélation des remplissages holocènes de vallons-secs en contexte loessique avec le profil sédimentaire synthétique du bassin de la Seine et avec le log synthétique d'une séquence de la vallée secondaire du Crould (95).

Si la Protohistoire semble marquer une rupture dans le fonctionnement des bassins versants [70], I'extension et l'ampleur des terrassements soumis aux travaux d'archéologie préventive, comme par exemple les travaux du contournement ouest de Strasbourg ou l'extension de la zone commerciale de Lampertheim au nord de Strasbourg, montrent que les remaniements contemporains sont absolument sans commune mesure avec les expressions sédimentaires protohistoriques et historiques. Leur quantification systématique apporterait des données locales complémentaires aux travaux en cours sur les faciès, les marqueurs géochimiques et les bilans sédimentaires de I'Anthropocène à partir du milieu du $X X^{e}$ siècle [71].

\section{CONCLUSION}

La démarche présentée ici, qui associe géomorphologues et archéologues, a connu un essor majeur grâce au développement de l'archéologie préventive à partir du milieu des années 1980 . Elle combine la réalisation de modèles de localisation et de conservation des sites, à partir de travaux de terrain et d'études documentaires, et les diagnostics archéologiques. Ancrée localement, adaptée au contexte topographique et géologique, elle concerne tous les acteurs de l'archéologie préventive hexagonale. Les exemples du Paléolithique aux périodes historiques présentés ici montrent qu'elle constitue à ce jour une méthode particulièrement adaptée pour trouver les sites archéologiques menacés par les travaux d'aménagement du territoire. Ces exemples illustrent également la possibilité d'écrire une géohistoire à partir de matériaux dont la collecte est imposée par une nécessité patrimoniale : sauver les sites archéologiques menacés par les travaux d'aménagement du territoire. Au final, « les disciplines des sciences de la terre sont, à part entière, des outils d'investigation du patrimoine archéologique » [72].

Si la démarche a jusqu'à présent surtout été appliquée avec succès à la sauvegarde et à l'étude des sites de la Préhistoire ancienne, elle ne l'est pas encore de façon uniforme sur l'ensemble du territoire. Elle gagnerait ainsi à être mise en œuvre plus systématiquement et mieux prise en compte lors des prescriptions, pour trouver les sites encore peu documentés,

[70] Voir par exemple ALLÉE \& LESPEZ 2006.

[71] Voir par exemple WATERS et al. 2018.

[72] KRIER 1994. 
comme par exemple les sites néolithiques, protohistoriques ou historiques stratifiés en fonds de vallées, notamment dans les vallées montagnardes ou dans les zones littorales. Il conviendrait également de mieux prendre en compte l'évolution récente des milieux et surtout la mécanisation des campagnes pour guider les diagnostics, mais aussi pour interpréter les résultats. Par ailleurs, une meilleure intégration des recherches géomorphologiques et géoarchéologiques dans les travaux archéologiques est souhaitable. L'organisation de séminaires régionaux réguliers associant chercheurs et acteurs du préventif serait une des façons d'y arriver, en parallèle des rencontres nationales et internationales déjà existantes. Le développement des outils géophysiques sur les fouilles archéologiques est également une piste prometteuse, si ces travaux intègrent les données pédologiques et sédimentaires collectées par les géoarchéologues [73]. Enfin, pour participer aux réflexions des communautés de chercheurs et pour développer des problématiques régionales, il ne faut pas oublier la nécessité de moyens humains suffisants et constants, pas toujours compatibles avec les tensions économiques d'une discipline minée par la mise en concurrence des différents opérateurs de l'archéologie préventive.

[73] Voir par exemple VELLA et al. 2013.

Allee, Philippe, Boumediene, Farid, Conte, Patrice, Diot, Marie-Françoise, Lehericy, Muriel, Petrt, Florent \& VALADA, Bernard, 2006, «Des archives sédimentaires aux dynamiques holocènes, l'exemple de deux études géoarchéologiques en Limousin », dans Philippe Allée \& Laurent Lespez (dir.), L'érosion entre société, climat et paléoenvironnement, Table ronde en l'honneur du Professeur René Neboit-Guilhot, Clermont-Ferrand 25-26-27 mars 2004, Clermont-Ferrand, p. 263-270.

Allee, Philippe, Diot, Marie-Françoise, Reynet, Jean-Michel \& Valadas, Bernard, 1997, « Trois mille ans d'enregistrement sédimentaire dans les fonds de vallons du Limousin », La dynamique des paysages protohistoriques, antiques, médiévaux et modernes, Sophia Antipolis, p. 365-387.

Allee, Philippe \& Lespez, Laurent, 2006, « De l'océanique au méditerranéen, la disparité des réponses morphosédimentaires holocènes, dans les massifs anciens européens », dans Philippe Allée \& Laurent Lespez (dir.), L'érosion entre société, climat et paléoenvironnement, Table ronde en I'honneur du Professeur René Neboit-Guilhot, Clermont-Ferrand 25-26-27 mars 2004, Clermont-Ferrand, p. 203-214.

Antorne, Philippe, Bahain, Jean-Jacques, Auguste, Philippe, Fagnard, Jean-Pierre, Limondin-Lozouet, Nicole \& LoCHT, Jean-Luc, 2011, «Quaternaire et préhistoire dans la vallée de la Somme : 150 ans d'histoire commune », dans Arnaud Hurel \& Noël Coye (dir.), Dans l'épaisseur du temps. Archéologues et géologues inventent la préhistoire, Paris, p. 341-381.

AuxietTe, Ginette \& Dubouloz, Jérôme, 2009, « Héritages d'une expérience trentenaire d'archéologie préventive dans la vallée de l'Aisne », dans Jean-Paul Demoule \& Christian Landes (dir.), La fabrique de l'archéologie en France, Paris, p. $147-162$. Bertran, Pascal, Sitzia, Luca, Banks, William E., Bateman, Mark D., Demars, Pierre-Yves, Hernandez, Marion, Lenorr, Michel, Mercier, Norbert \& Prodeo, Frédéric, 2013, « The Landes de Gascogne (southwest France): periglacial desert and cultural frontier during the Palaeolithic », Journal of Archaeological Science, 40/5, p. $2274-2285$.

BlondeAU, Cécile, 2018, Oberhergheim (Haut-Rhin) : "Oberfeld », rue de Rouffach, rapport de diagnostic, Sélestat.

Blondeau, Cécile \& Wuscher, Patrice, 2017, Ruelisheim (Haut-Rhin) : lotissement « Les Jonquilles » : Une occupation Néolithique et Bronze ancien, Sélestat.

Borderie, Quentin, Chamaux, Gabriel, Kreutzer, Sebastian \& Ahmed-Delacroix, Eric Nelson, 2019, « Occupations humaines et chronstratigraphie du gisement pléistocène d'Illiers-Combray (Eure-et-Loir, France) : de nouveaux éléments pour le SIM 5b », Bulletin de la Société Préhistorique Française 116/1, p. 7-28.

Borderie, Quentin, Chamaux, Gabriel, Roussaffa, Hugo, Douard, Michel, Fencke, Emilie, Rodot, MarieAngélique, Perrichon, Pierre \& Selles, Hervé, 2017, « La couverture loessique d'Eure-et-Loir (France) : potentiel pédo-sédimentaire et organisation spatiale ; objectifs, méthodes et premiers résultats du programme QuOrEL », Quaternaire 28/3, p. 389-400.

Bravard, Jean-Paul, Burnouf, Joëlle \& Verot, Agnès, 1989, « Géomorphologie et archéologie dans la région lyonnaise : questions et réponses d'un dialogue interdisciplinaire », Bulletin de la Société préhistorique française 86/10, p. 429-440. BRUXELLeS, Laurent, 2010, « Diagnostic archéologique et géomorphologie en Midi-Pyrénées : réflexions méthodologiques concernant la recherche de vestiges paléolithiques », dans Pascal Depaepe \& Frédéric Séara (éd.), Le diagnostic des sites paléolithiques et mésolithiques, actes du séminaire des 5 et 6 décembre 2006, Paris, p. 88-95. 
BRUXELles, Laurent, 2012, «De la contribution à la synergie : une décennie de relations archéologues-géomorphologues à I'Inrap », Archéopages Hors série 3, p. 21-25.

Bruxelles, Laurent, Berthet, Anne-Laure, Chalard, Pierre, Colonge, David, Delfour, Géraldine, JarRy, Marc, Lelouvier, Laure-Amélie, ARnouX, Thomas \& ONezIME, Olivier, 2003, « Le paléolithique inférieur et moyen en Midi toulousain : nouvelles données et perspectives de l'archéologie préventive », Paléo 15, p. 7-28.

BruXelles, Laurent \& JARRY, Marc, 2011, « Climatic conditions, settlement patterns and cultures in the Paleolithic: The example of the Garonne Valley (southwest France) », Journal of Human Evolution 61/5, p. 538-548.

CAStanet, Cyril, 2008, La Loire en val d'Orléans : dynamiques fluviales et socio-environnementales durant les derniers 30000 ans : de l'hydrosystème à l'anthroposystème, thèse de doctorat, Paris.

Chausse, Christine, 2003, Les nappes alluviales de la basse vallée de l'Yonne, approche géométrique et chronostratigraphique et l'apport de l'étude de la Nappe de Soucy à la compréhension des occupations du Paléolithique inférieur de Soucy, thèse de doctorat, Lille.

Cloots, Anne-Rose, Maire, Gérard, Schneider, Charles, Carado, Annick \& Tricart, Jean, 1973, Carte géomorphologique détaillée de la France 1:50, 000. XXXVIII-16, Strasbourg, Paris.

ColLART, Jean-Luc, 2012, «L'archéologie préventive en France : le cadre réglementaire et son application », Developmentled Archaeology in Northwest Europe, Proceedings of a Round Table at the University of Leicester 19th-21st November 2009, Leicester, p. 56-99.

Colonge, David, Arnoux, Thomas, Bruxelles, Laurent, Chevreuse, Fabrice, D'Aggostino, Antonio, Jamois, Marie-Hélène \& ONezIME, Olivier, 2004, Aérodrome de Brive - Souillac communes de Nespouls (Corrèze, Limousin) Cressensac (Lot, Midi-Pyrénées), Rapport final d'opérations de sondages de diagnostic et évaluations, Rapport de diagnostic, Pessac.

Confalonieri, Joël \& Le Jeune, Yann, 2012, « Le site mésolithique de la Haute-Ile à Neuilly-sur-Marne (Seine-SaintDenis) : premiers résultats », dans Boris Valentin, Bénédicte Souffi, Ducrocq Thierry (éd.), Palethnographie du Mésolithique. Recherches sur les habitats de plein air entre Loire et Neckar Actes de la table ronde de Paris, 26 et 27 novembre 2010, p. 51-67. Croutsch, Christophe, 2019, Eckwersheim « Lotissement auf die Niedermatten », Rapport de diagnostic, Sélestat.

DAUgAs, Jean-Pierre \& Bonin, Thierry (éd.), 2006, Le diagnostic archéologique en milieu rural, actes du séminaire de Glux-en-Glenne 25-27 octobre 2005, Paris.

Daveau, Isabelle, 2007, Port Ariane : Occupations et utilisation d'une zone humide lors des six derniers millénaires à Lattes (Hérault), Lattes.

Depaepe, Pascal \& Seara, Frédéric (éd.), 2010, Le diagnostic des sites paléolithiques et mésolithiques, actes du séminaire des 5 et 6 décembre 2006, Paris.

DESCHODT, Laurent, 2010, «L'exemple d'une campagne de sondages géologiques préalables au diagnostic archéologique », dans Pascal Depaepe \& Frédéric Séara (éd.), Le diagnostic des sites paléolithiques et mésolithiques, actes du séminaire des 5 et 6 décembre 2006, Paris, p. 8-12.

DeschodT, Laurent, 2014, Chronostratigraphie et paléoenvironnements des fonds de vallée du bassin français de l'Escaut, thèse de doctorat, Paris.

Duвouloz, Jérôme, 2003, «L'évaluation des méthodes de diagnostic : simulations sur des sites de l'Aisne », Les Nouvelles de l'Archéologie 91, p. 46-50.

DucrocQ, Thierry, 2010, « La détection des sites mésolithiques dans le nord de la France », dans Pascal Depaepe \& Frédéric Séara (éd.), Le diagnostic des sites paléolithiques et mésolithiques, actes du séminaire des 5 et 6 décembre 2006, Paris, p. 30-34. Elsass, Philippe, Huggenberger, Peter \& Wirsing, Gunther, 2006, Structure hydrogéologique et caractéristiques hydrauliques, INTERREG III A : MoNit « Modélisation de la pollution des eaux souterraines par les nitrates dans la vallée du Rhin Supérieur », Karlsruhe.

Ertlen, Damien, Gebhardt, Anne, Schneider, Nathalie, Durand, Frédérique, Thomas, Yohann, Michler, Matthieu, SCHNeIKeRT, François, Bö̈s, Eric \& SChWARTZ, Dominique, 2012, « Anthropisation et érosion agraire dans un paysage lœssique (Bas-Rhin, France) », dans Frédéric Bertoncello \& Frank Braemer (dir.), Variabilités environnementales, mutations sociales. Nature, intensités, échelles et temporalités des changements, XXXIIe rencontres internationales d'archéologie et d'histoire d'Antibes, Antibes, p. 85-92.

FORRER, Robert, 1925, Les éléphants, hippopotames et I'homme de l'Alsace quaternaire: étude de géographie paléolithique régionale, Colmar.

GuILHOT, Jean-Olivier, 2018, Bilan sur la mise en place des commissions territoriales de la recherche archéologique (CTRA) et Rapports d'activité 2017 des CTRA, Paris.

Hurel, Arnaud \& CoYe, Noël, 2011, Dans l'épaisseur du temps. Archéologues et géologues inventent la préhistoire, Paris. Jung, Cécile, Jorda, Christophe, Rascalou, Pierre \& Sendra, Benoît, 2017, Céreirède Maera (Lattes, Hérault), Rapport de diagnostic, Nîmes.

KRIER, Vincent, 1994, «Les disciplines des sciences de la terre et l'archéologie de sauvetage : leçons d'une expérience », Les Nouvelles de I'Archéologie 58, p. 43-44.

KRIER, Vincent, 2004, La plaine alluviale de I'Oise : milieu et système fluvial ; stratigraphie des formations alluviales et archéologie, Saint-Ouen-I'Aumone.

Lebret, Patrick \& HAlbout, Hervé, 1991, Le quaternaire dans le Val d'Oise, Caen.

Locht, Jean-Luc, Sellier, Nathalie, Coutard, Sylvie, Antoine, Pierre \& Feray, Philippe, 2010, « La détection de sites du Paléolithique ancien et moyen dans le nord de la France : approche particulière », dans Pascal Depaepe \& Frédéric Séara (éd.), Le diagnostic des sites paléolithiques et mésolithiques, actes du séminaire des 5 et 6 décembre 2006, Paris, p. 49-57.

MALRAIN, François, 2008, « Silence au fond des vallées », Constructions de l'archéologie, Paris, p. 119-120. 
MARMet, Eric, Best, Christine \& TABbagh, Alain, 2002, «Prospection systématique par sondages à la pelle mécanique : limites liées à la probabilité de découverte de sites archéologiques », ArchéoSciences, revue d'Archéométrie 26/1, p. 11-21. Marsac, Romain, Ard, Vincent, Bolo, Aurélien, Duschesnes, Sylvie, Fabre, Magali, Farge, Antoine, Forest, Vianney, Gandelin, Muriel, Gleize, Yves, Hamon, Caroline, Jorda, Christophe, Laurent, Amélie, Lea, Vanessa, Martin, Sophie, Mens, Emmanuel, Vinolas, Frédéric \& Wattez, Julia, 2019, Ligne Grande Vitesse Contournement de Nîmes et Montpellier, Secteur 3 (Lattes, Hérault), La Céreirède-Rauze Basse. Un monument funéraire du Chasséen récent, Rapport Final d'Opération (fouille préventive), Montpellier.

Moine, Olivier, Wuscher, Patrice, Krauss, Lydia, TAYlor, Samuel, Villa, Valentina \& Zens, Jörg, 2017, « Séries loessiques, préhistoire et variabilité environnementale millénaire des deux dernières périodes glaciaires à Achenheim », Le Quaternaire du Rhin supérieur, France, Allemagne et Suisse. Livret-guide de l'excursion Afeq CNF-INQUA 17-19 mai 2017, Sélestat, p. 33-39.

MoRdant, Daniel, 1994, «L'archéologie en gravières vue à travers l'exemple de la Bassée », Les Nouvelles de l'Archéologie 58, p. 36-40.

OTt Matthieu \& TARrou Liliane, 2013, Doublement de l'autoroute A9, contournement de Montpellier, secteur 2 (Lattes, Hérault), Rapport de diagnostic.

PASTRE Jean-François, 1994, «Les recherches géomorphologiques et paléo-environnementales liées à l'archéologie de sauvetage : quelques vues d'ensemble », Les Nouvelles de l'Archéologie 58, p. 41-42.

Pastre, Jean-François, Leroyer, Chantal, Limondin-Lozouet, Nicole, Antoine, Pierre, Chausse, Christine, Gauthier, Agnès, Granai, Salomé, Le Jeune, Yann \& Wuscher, Patrice, 2014, « L'Holocène du Bassin parisien (France), apports de l'étude géoécologique et géoarchéologique des fonds de vallées », dans Nathalie Carcaud \& Gilles Arnaud-Fassetta (dir.), La géoarchéologie française au XXI siècle, Paris.

PezIn, Annie, 2014, Amélie-les-Bains, Camp de las Basses, entre fleuve et versant, de l'âge du Bronze à l'Antiquité, rapport de fouille préventive, Nîmes.

Pezin, Annie \& Wuscher, Patrice, 2005, Amélie-les-Bains (Pyrénées-Orientales), Camps de las Basses : occupations humaines de l'âge du Bronze à l'époque moderne, entre fleuve et versant, en moyenne vallée du Tech, Rapport de diagnostic, Montpellier.

Pierrevelcin, Gilles, Bonnaire, Emmanuelle, Higelin, Mathias, Leprovost, Céline, Pascutto, Émilie, Putelat, Olivier, Roth-Zehner, Muriel \& Wuscher, Patrice, 2016, Ensisheim (68), Reguisheimer Feld/RD201 tranche 2 : Un nouvel habitat de La Tène finale à Ensisheim «Reguisheimer Feld», rapport de fouille préventive, Sélestat. Pomerol, Charles, Bricon, Claude \& Guernet, Claude, 1969, Carte géologique détaillée de la France, Étampes, Orléans. Rascalou, Pierre \& Bel, Valérie, 2013, Ligne Grande Vitesse Contournement de Nîmes et Montpellier, Secteur 3 (Lattes, Hérault), Rapport de diagnostic, Nîmes.

RauX, André, Bel, Valérie, Jorda, Christophe \& Sejalon, Pierre, 2013, Doublement de l'autoroute A9, contournement de Montpellier, secteur 3 (Lattes, Hérault), Rapport de diagnostic, Nîmes.

Roth-Zehner, Muriel, Bachellerie, François \& Wuscher, Patrice, 2016, Ensisheim (68) : « Reguisheimer Feld », ZAID de Ensisheim/Réguisheim [tranche 3], rapport de diagnostic, Sélestat.

Roth-Zehner, Muriel, Delloul, Marion, Griselin, Sylvain, LeProvost, Céline, Rault, Estelle \& Wuscher, Patrice, 2019, Ensisheim, Haut-Rhin, ZAID de Ensisheim / Réguisheim : Tranche 4, Reguisheimer Feld, rapport de diagnostic, Sélestat.

Schneider, Nathalie \& ErTlen, Damien, 2017, Géoarchéologie, des Vosges au Kochersberg : les données de diagnostic et de fouilles de la Ligne à Grande Vitesse Est Européenne (LGVEE). Rapport annuel du projet «Empreintes de I'Homme dans I'Environnement, entre Vosges et Rhin », Strasbourg.

Schneider, Nathalie, Ertlen, Damien, Durand, Frédérique, Nocus, Noémie, Gebhardt, Anne, Thomas, Yohann, MichleR, Matthieu, Schnerkert, François \& BoËs, Éric, 2014, « Diagnostic LGV Est Européenne en Alsace (France). Suivi géoarchéologique et études paléoenvironnementales : les clés de lecture du paysage », dans Nathalie Carcaud \& Gilles Arnaud-Fassetta (dir.), La géoarchéologie française au XXI ${ }^{e}$ siècle, Paris, p. 35-45.

SChNeIKeRT, François, 2017, Contournement Ouest de Strasbourg, Tronçon 2. Achenheim, Breuschwickersheim, Ernolsheim-sur-Bruche, Ittenheim, Kolbsheim, Osthoffen (Bas-Rhin) : A355, rapport de diagnostic, Sélestat.

Schneikert, François \& Wuscher, Patrice, 2016, Pulversheim (68) : "Ochsenweidle », rue de Cernay / rue de Mulhouse, rapport de diagnostic, Sélestat.

Souffi, Bénédicte, BlASER, Frédéric, VAlentin, Boris, 2009, Paléolithique et Mésolithique en régions Centre et Ile-de-France : modalités d'implantation et de conservation des sites, rapport de projet d'activité scientifique (PAS), INRAP. Megnien, François, 1987, Carte géologique de la France à 1:50 000, Corbeil-Essonnes, Orléans.

Vacchi, Matteo, Marriner, Nick, Morhange, Christophe, Spada, Giorgio, Fontana, Alessandro \& Rovere, Alessio, 2016, « Multiproxy assessment of Holocene relative sea-level changes in the western Mediterranean: Sea-level variability and improvements in the definition of the isostatic signal », Earth-Science Reviews 155, p. 172-197.

Vella, Marc-Antoine, Ghilardi, Matthieu, Diouf Ousmane, Parisot, Jean-Claude, Hermitte, Daniel, Provansal, Mireille, Fleury, Jules, Dussouillez, Philippe, Delanghe-Sabatier, Doriane, Demory, François, Quesnel, Yoann, HARTMAnN-Virnich, Andreas, Delpey, Yhann, Berthelot, Michel, Bicket, Andrew, 2013, « Géoarchéologie du Rhône dans le secteur du pont Saint-Bénézet (Avignon, Provence, France) au cours de la seconde moitié du deuxième millénaire apr. J.-C. : étude croisée de géographie historique et des paléoenvironnements », Géomorphologie : relief, processus, environnement 19/3, p. 287-310.

VoGT, Henri, 1992, Le relief en Alsace : étude géomorphologique du rebord sud-occidental du Fossé rhénan, Strasbourg. Waters, Colin N., Fairchild, Ian J., McCarthy, Francine M. G., Turney, Chris S. M., Zalasiewicz, Jan \& WILliamS, Mark, 2018, « How to date natural archives of the Anthropocene », Geology Today 34/5, p. $182-187$. 
Wuscher, Patrice, Bachellerie, François, Koehler, Héloïse, Diemer, Simon, Griselin, Sylvain, Goudissard, Simon, Feliu, Clément, Schneider, Nathalie, Pracht, Axel, Fabre, Magali, Moine, Olivier, Claud, Emilie, Salomon, Ferréol, Rambeau, Claire, Schmitt, Laurent, Seveque, Noémie, Preusser, Frank, Antoine, Pierre, Rinterknecht, Vincent, Villa, Valentina, Rixhon, Gilles, Bö̈s, Eric, Basoge, florian \& Schwartz, Dominique, 2019, Le Paléolithique et le Mésolithique de la Plaine d'Alsace et des collines sous-vosgiennes : reprise des données existantes, pistes pour détecter les sites et approches territoriales. Bilan de l'année 2018, Strasbourg.

Wuscher, Patrice, Blaser, Frédéric, Blaser, Romana, Rouppert, Vanessa, Granchon, Philippe, Jobic, Françoise, Bayle, Grégory, Delattre, Valérie, Du BouËtiez de Kerorguen, Emmanuelle \& LeConte, Luc, 2013, Le Thillay « les Grands Champs » (Ile-de-France, Val-d'Oise), Rapport de diagnostic, La Courneuve.

Wuscher Patrice, Blaser Romana, Cammas Cécilia, Durand Juliette, Glisoni Steve, Saron Emmanuelle \& PASTRE Jean-François, 2012, «Des plateaux, des limons et des hommes : état des connaissances et questions sur les sols et l'érosion tardiglaciaires et holocènes en Ile-de-France », Revue Archéologique d'Ile-de-France 5, p. 5-28.

Wuscher, Patrice, Diemer, Simon, Koehler, Héloöse, Bachellerie, François, Griselin, Sylvain, Goudissard, Simon, Schneider, Nathalie, Seveque, Noémie, Basoge, Florian, Preusser, Frank, Moine, Olivier, Boës, Eric, Pracht, Axel, Detrey, Jean, Lefranc, Philippe, Ertlen, Damien \& Affolter, Jehan, 2018, Le Paléolithique et le Mésolithique de la Plaine d'Alsace et des collines sous-vosgiennes : reprise des données existantes, pistes pour détecter les sites et approches territoriales. Bilan de l'année 2017, rapport de Projet Collectif de Recherche, Strasbourg.

Wuscher, Patrice \& Pezin, Annie, 2010, « Morphogenèse de la vallée du Tech à Amélie-les-Bains (Pyrénées-Orientales) durant l'Holocène et évolution des versants jusqu'à la fin de l'âge du Bronze », Quaternaire 21/4, p. 345-356. 